Systematic Review

\title{
Treatment of Chemotherapy-Induced Peripheral Neuropathy: Systematic Review and Recommendations
}

Saiyun Hou, MD, PhD'1, Billy Huh, MD, PhD' ${ }^{1}$, Hee Kee Kim, PhD', Kyung-Hoon Kim, MD, PhD², and Salahadin Abdi, MD, PhD ${ }^{1}$

From: ${ }^{1}$ Department of Pain Medicine, The University of Texas MD Anderson Cancer Center, Houston, TX 77030

${ }^{2}$ Pusan National University, Pusan, S. Korea

Address Correspondence: Salahadin Abdi, MD, PhD Department of Pain Medicine

The University of Texas MD Anderson Cancer Center 1400 Holcombe Blvd, Unit 0409 Houston, TX 77030'

E-mail: sabdi@mdanderson.org

Disclaimer: See P. 589 Conflict of interest: Each author certifies that he or she, or a member of his or her immediate

family, has no commercial association (i.e., consultancies, stock ownership, equity interest, patent/licensing arrangements, etc.) that might pose a conflict of interest in connection with the submitted manuscript.

Manuscript received: $04-10-2018$ Revised manuscript received: o6-14-2018

Accepted for publication: 07-19-2018

Free full manuscript: www.painphysicianjournal.com
Background: Chemotherapy-induced peripheral neuropathy (CIPN) is a commonly encountered disease entity following chemotherapy for cancer treatment. Although only duloxetine is recommended by the American Society of Clinical Oncology (ASCO) for the treatment of CIPN in 2014, the evidence of the clinical outcome for new pharmaceutic therapies and non-pharmaceutic treatments has not been clearly determined.

Objective: To provide a comprehensive review and evidence-based recommendations on the treatment of CIPN

Study Design: A systematic review of each treatment regimen in patients with CIPN.

Methods: The literature on the treatment of CIPN published from 1990 to 2017 was searched and reviewed. The 2011 American Academy of Neurology Clinical Practice Guidelines Process Manual was used to grade the evidence and risk of bias. We reviewed and updated the recommendations of the ASCO in 2014, and evaluated new approaches for treating CIPN

Results: A total of 26 treatment options in 35 studies were identified. Among these, 7 successful RCTs, 6 failed RCTs, 18 prospective studies, and 4 retrospective studies were included. The included studies examined not only pharmacologic therapy but also other modalities, including laser therapy, scrambler therapy, magnetic field therapy and acupuncture, etc. Most of the included studies had small sample sizes, and short follow-up periods. Primary outcome measures were highly variable across the included studies. No studies were prematurely closed owing to its adverse effects.

Limitations: The limitations of this systematic review included relatively poor homogeneous, with variations in timing of treatment, primary outcomes, and chemotherapeutic agents used.

Conclusion: The evidence is considered of moderate benefit for duloxetine. Photobiomodulation, known as low level laser therapy, is considered of moderate benefit based on the evidence review. Evidence did not support the use of lamotrigine and topical KA (4\% ketamine and 2\% amitriptyline). The evidence for tricyclic antidepressants was inconclusive as amitriptyline showed no benefit but nortriptyline had insufficient evidence. Further research on CIPN treatment is needed with larger sample sizes, long-term follow-up, standardized outcome measurements, and standardized treatment timing.

Key words: Chemotherapy-induced neuropathy, peripheral neuropathy, chemotherapy-tumor, neuropathic pain, chronic pain, toxicology, treatment, reduction of pain, level of evidence.

Pain Physician 2018: 21:571-592 hemotherapy-induced peripheral neuropathy (CIPN) is a common side effect of neurotoxic Jchemotherapeutic agents such as taxanes, vinca alkaloids, platinum compounds, bortezomib, and thalidomide. CIPN is mainly involved in a sensory peripheral neuropathy though some patients experience motor symptoms such as weakness and autonomic neuropathy (1). The sensory neuropathy is 
often distributed in a "stocking and glove" manner, causing symptoms such as pain, allodynia, loss of sensation, paresthesia, numbness, tingling, and gait disturbance $(2,3)$. CIPN can cause significant loss of functional abilities and negatively affect quality of life, which can lead to dose reductions, discontinuation of treatment, and ultimately affect overall survival (2). CIPN has dose limiting side effect that likely will increase in prevalence as new cancer therapies extend patient survival times. One study established the prevalence of CIPN at $68.1 \%$ in the first month after chemotherapy, $60.0 \%$ at 3 months, and $30.0 \%$ at 6 months or more (1). Some chemotherapy drugs were associated with even higher prevalence and duration of CIPN. For instance, approximately $80 \%$ of patients who received taxanes and oxaliplatin therapy continue to suffer from CIPN even 6 months or 2 years after chemotherapy (4-6).

At present, no sufficient treatment options are available for CIPN and its exact pathophysiology is not clear. CIPN is most commonly considered as neuropathic pain due to axonopathy by dying back axonal degeneration (7). However, most of the pharmacologic treatments for neuropathic pain including tricyclic antidepressants, and anticonvulsants, are minimally effective in CIPN or have unacceptable side effects (8-11). Therefore, the mechanisms underlying CIPN may differ from those involved in typical neuropathic pain conditions.

To date only duloxetine is recommended by the American Society of Clinical Oncology (ASCO) for the treatment of CIPN, on the bases of a modest positive result in 1 randomized control trial (RCT) (12). In addition, several systemic reviews (12-15) evaluating strategies for the prevention and treatment of CIPN have been recently published; however these reviews focus only on pharmacologic treatments of CIPN and were mainly limited to RCTs. Here we performed the evaluation of all selected studies, not only of pharmacologic treatments, but also of non-pharmacologic treatment modalities. The goals of this systematic review were to 1) provide a comprehensive systematic review for all available treatments for CIPN; 2) evaluate the strengths and weaknesses of each study; and 3) summarize the level of evidence and provide evidence-based recommendations.

\section{Methods}

The methodology used in this systematic review followed the procedures for systematic reviews of therapeutic questions in the 2011 American Academy of Neurology (AAN) Clinical Practice Guideline Process Manual $(16,17)$. We chose to use the AAN Clinical Practice Guideline Process Manual for this review because it formed the basis of 45 evidence-based reviews published by the AAN and made available on the Agency for Healthcare Research and Quality's National Guideline Clearinghouse (www.guideline.gov), a public resource for evidence-based clinical practice guidelines (16-18). It has been widely used by many authors (19-22).

\section{Criteria for Considering Studies for this Review}

\section{Types of Studies}

RCTs and prospective non-randomized, case-control, cohort, and cross-over studies, and retrospective studies published in English from January, 1990 to September, 2017 were included. Case reports, case series, abstracts, book chapter, and review articles, letters to the editor, and newspaper articles were excluded. Animal studies and studies of children were also excluded.

\section{Types of Participants}

Patients of interest were adults with CIPN of at least 18 years of age.

\section{Types of Interventions}

Studies evaluating any treatment modality, including pharmacologic and non-pharmacologic modalities, were included.

\section{Types of Outcome Measures}

Outcome measures are variable in all selected studies so that the uses of specific outcome measures were not inclusion criteria. Here we mainly focus on pain relief and the change in severity of neuropathic symptoms as the primary outcome parameter.

\section{Literature Search}

All of the available trials or studies in any language from any country describing appropriate management of CIPN with outcome evaluations were considered for inclusion. We searched Pubmed, Scopus, the Cochrane Library, and ClinicalTrials.gov for studies published from January 1990 through September 2017.

The search strategy was designed to identify studies of the treatment of CIPN. The search terms included: "chemotherapy," "peripheral neuropathy," "neuro- 
pathic pain," "treatment," and "cancer." Different combinations of the search terms were made by using the Boolean operators "AND," "OR," and "NOT."

\section{Data Extraction}

Two review authors working independently, in a non-blinded standardized manner, searched for relevant literature, and extracted the data from the included studies. Disagreements were resolved by discussion between the 2 authors; if no agreement could be reached, another resolved the dispute.

\section{Analysis of Evidence}

The quality of the included studies' evidence was analyzed using the methods described in the 2011 AAN Clinical Practice Guideline Process Manual $(16,17)$. Studies were first classified on the basis of the strength of the evidence they presented into 4 levels ranging from the strongest, Class I, to the weakest, Class IV (Table 1). The level of confidence in the evidence was determined using 4 levels of confidence, as described in the 2011 AAN Clinical Practice Guideline Process Manual (Table 2). Level of confidence in evidence is initially

Table 1. Rating scheme for the strength of evidence for therapeutic questions.

\begin{tabular}{|c|c|}
\hline Class I & $\begin{array}{l}\text { Randomized, controlled clinical trial (RCT) in a representation population } \\
\text { Masked or objective outcome assessment } \\
\text { Relevant baseline characteristics are presented and substantially equivalent between treatment groups, or there is appropriate } \\
\text { statistical adjustment for differences } \\
\text { Also required: } \\
\text { Concealed allocation } \\
\text { No more than } 2 \text { primary outcomes specified } \\
\text { Exclusion/inclusion criteria clearly defined } \\
\text { Adequate accounting for dropouts (with at least } 80 \text { percent of enrolled subjects completing the study) and crossovers with } \\
\text { numbers sufficiently low to have minimal potential for bias } \\
\text { For noninferiority or equivalence trials claiming to prove efficacy for } 1 \text { or both drugs, the following are also required * } \\
\text { The authors explicitly state the clinically meaningful difference to be excluded by defining the threshold for equivalence or } \\
\text { noninferiority } \\
\text { The standard treatment used in the study is substantially similar to that used in previous studies establishing efficacy of the } \\
\text { standard treatment (e.g., for a drug, the mode of administration, dose, and dosage adjustments are similar to those previously } \\
\text { shown to be effective) } \\
\text { The inclusion and exclusion criteria for patient selection and the outcomes of patients on the standard treatment are } \\
\text { comparable to those of previous studies establishing efficacy of the standard treatment } \\
\text { The interpretation of the study results is based on a peer-protocol analysis that accounts for dropouts or crossovers. } \\
\text { For crossover trials, both period and carryover effects examined and statistical adjustments performed, if appropriate }\end{array}$ \\
\hline Class II & $\begin{array}{l}\text { An RCT that lacks } 1 \text { or } 2 \text { criteria a-e (see Class I) or a cohort study meeting criteria b-e (see Class I) } \\
\text { Randomized, crossover trial missing } 1 \text { of the following } 2 \text { criteria: } \\
\text { Period and carryover effects described } \\
\text { Baseline characteristics of treatment order groups presented } \\
\text { All relevant baseline characteristics are presented and substantially equivalent among treatment groups, or there is } \\
\text { appropriate statistical adjustment for differences } \\
\text { Masked or objective outcome assessment }\end{array}$ \\
\hline Class III & $\begin{array}{l}\text { Controlled studies (including studies with external controls such as well-defined natural history controls) } \\
\text { Crossover trial missing both of the following } 2 \text { criteria: } \\
\text { Period and carryover effects } \\
\text { Baseline characteristics presented } \\
\text { A description of major confounding differences between treatment groups that could affect outcome } \\
\text { Outcome assessment masked, objective, or performed by someone who is not a member of the treatment team }\end{array}$ \\
\hline Class IV & $\begin{array}{l}\text { Did not include patients with the disease } \\
\text { Did not include patients receiving different interventions } \\
\text { Undefined or unaccepted interventions or outcome measures } \\
\text { No measures of effectiveness or statistical precision presented or calculable }\end{array}$ \\
\hline
\end{tabular}

${ }^{*}$ Numbers $1-3$ in Class I are required for Class II in equivalence trials. If any 1 of the 3 is missing, the class is automatically downgraded to Class III

${ }^{* *}$ Objective outcome measurement: An outcome measure that is unlikely to be affected by an observer's (patient, treating physician, investigator) expectation or bias (e.g., blood tests, administrative outcome data) . 
anchored to class of evidence; then considering various factors that can downgrade or upgrade confidence in evidence, which was described previously $(16,17)$.

The strength of the recommendations for use of the therapeutic modalities was determined on basis of the level of confidence in the evidence. Recommendation strength was stratified into 4 levels: $A$, clinicians must or must not offer the treatment; B, clinicians should or should not offer the treatment; $C$, clinicians may or may not offer the treatment; and $U$, no recommendation (Table 3) $(16,17)$. Other factors that affected the recommendation strength included: the generalizability of the study, the clinical importance of the treatment's effect, the risk of harm weighed against the benefit of the treatment, the treatment availability and cost, and the alternative interventions.

\section{REsULts}

\section{Search Results}

A total of 1,288 relevant studies were identified by the literature search. Of these, 117 studies were examined in detail and a total of 35 studies ultimately met all inclusion criteria $(3,8-11,23-52)$. The included studies described total 26 treatment options for CIPN, including pharmacological therapy, light therapy, scrambler therapy, magnetic field therapy, acupuncture, dietary therapy and long-wave diathermy therapy, etc. Among included studies, 7 successful RCTs, 6 failed RCTs, 18 prospective studies, and 4 retrospective studies were identified (Fig. 1).

\section{Quality Assessment}

Table 4 described the results of each study reviewed. The strengths and weaknesses of each study were evaluated and quality assessment is summarized in Table 5. On the basis of the AAN criteria, the level of confidence in the evidence was determined for each treatment options. But there are various factors that can downgrade (e.g., power) or upgrade confidence in evidence $(16,17)$. Based on the strengths and weaknesses for each study, the level of confidence in evidence for each treatment option was summarized in Table 6. Among 7 successful RCTs, duloxetine had Class II level of strength of evidence and photobiomodulation had Class I, but downgraded to Class II due to relative small sample size. The remaining 5 studies were identified as 2 Class IV and 3 Class II initially, then downgraded to very low level of confidence in evidence due to poor design quality. In 6 failed RCTs, 2 Class II large sample size studies with treatment of lamotrigine, and topical $2 \%$ amitriptylin/ 4\% ketamine showed negative outcome. Other studies presented with poor design quality with Class IV evidence level.

\section{Recommendations}

The strength of the recommendation for each treatment option is shown in Table 6. The majority of treatments were considered level $U$ (no recommendation) on the basis of a paucity of high-quality and consistent evidence. No recommendation can be made for the following treatments:

1. Acetyl-L-carnitine (ALC)

2. Amitriptyline

Table 2. Level of confidence in evidence.

\begin{tabular}{|l||}
\hline - High confidence corresponds to a "highly likely" conclusion; anchor is 2 Class I studies \\
\hline - Moderate confidence corresponds to "likely" conclusion; anchor is 1 Class I study or 2 Class II studies \\
\hline - Low Confidence corresponds to "possibly" conclusion; anchor is 1 Class II study, or 2 Class III studies \\
\hline - Very low confidence corresponds to "insufficient" conclusion; anchor is < 2 Class III \\
\hline \hline
\end{tabular}

Table 3. Rating scheme for the strength of the recommendations.

\begin{tabular}{|l|l||}
\hline Classification of recommendations \\
\hline Level A & $\begin{array}{l}\text { Strongest recommendation, and is denoted by "must" or "must not"; based on high confidence in the evidence, and high } \\
\text { magnitude of benefit and low risk; "must" recommendations are relatively rare }\end{array}$ \\
\hline Level B & $\begin{array}{l}\text { "Should" or "should not"; tend to be more common than A, since requirements are less stringent but still based on moderate } \\
\text { evidence and risk/benefit profile }\end{array}$ \\
\hline Level C & "May" or "may not"; lowest allowable recommendation \\
\hline Level U & No recommendation can be made because of insufficient evidence \\
\hline
\end{tabular}




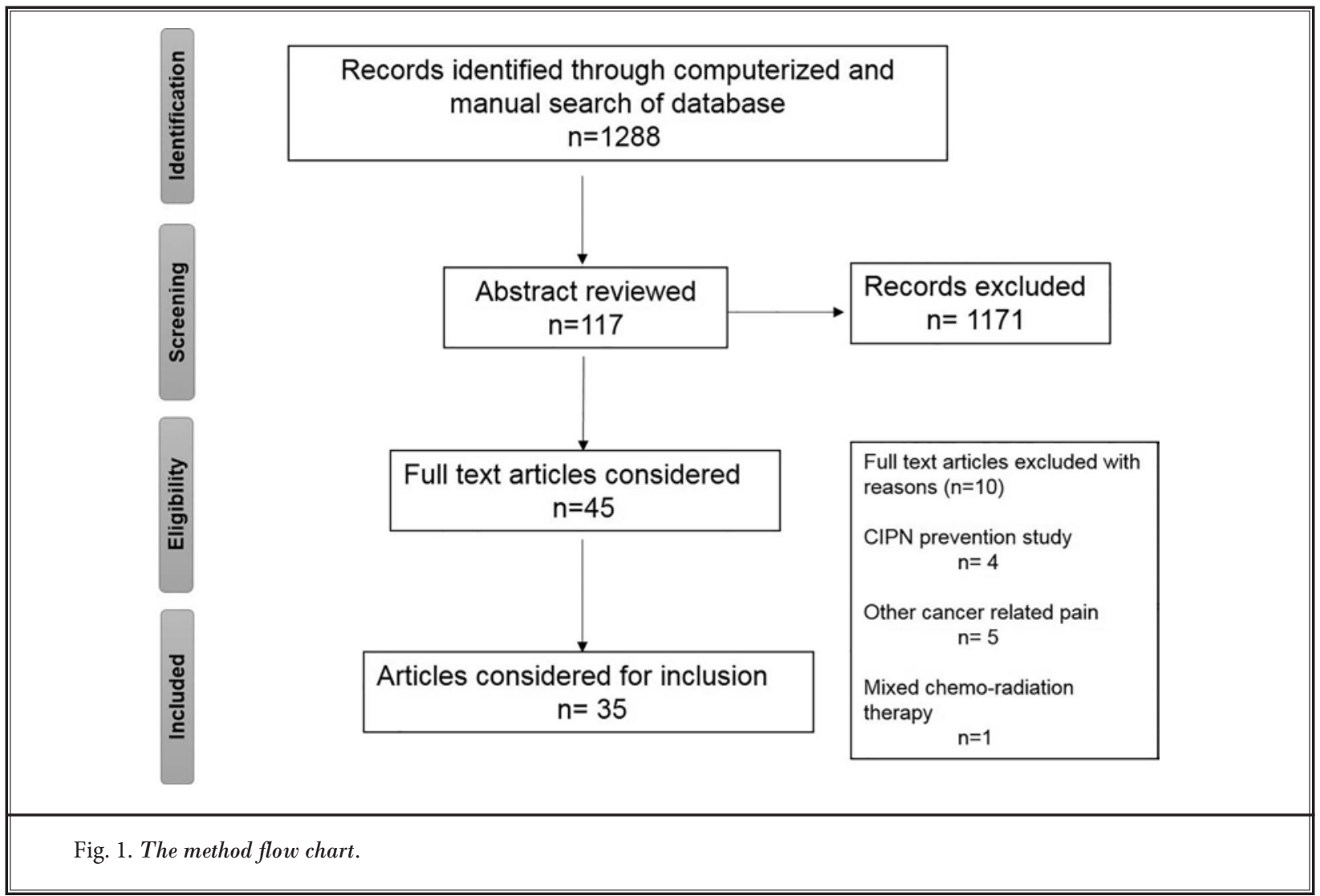

3. Cannabinoids

4. Gabapentin

5. Intravenous lidocaine

6. Neurofeedback (NFB)

7. Nortriptyline

8. Alpha lipoic acid, methylsulfonylmethane, and bromelain dietary supplement (OPERA)

9. Oxycodone (long-acting)

10. Palmitoylethanolamide (PEA)

11. Venlafaxine

12. Topical baclofen, amitriptyline and ketamine (BAK-PLO)

13. Topical menthol (TRPM8 agonist)

14. Acupuncture

15. Acupuncture like transcutaneous nerve stimulation (ALTENS)

16. Electro-acupuncture

17. Laser acupuncture (LA)

18. Percutaneous auricular neurostimulation (PANS)

19. Sweet bee venom pharmacopuncture (SBVP)

20. Interferential therapy and long-wave diathermy at high power (ITH)
21. Low-frequency magnetic field therapy

22. Scrambler therapy

Lamotrigine and topical KA (4\% ketamine and 2\% amitriptyline) should not be offered by physicians for treating CIPN, as they appear to have no benefit even in a large RCT study and many severe adverse events (AEs) in topical KA (35).

To date, only Duloxetine and photobiomodulation (PBM) can be considered to provide a modest benefit for patients with CIPN. Physicians may offer them for patients with cancer experiencing CIPN. PBM, a low level laser therapy, showed significant improvement in modified total neuropathy score (mTNS) at 8 weeks. Although this was a high-quality study with consistent evidence, we downgraded to the low level of confidence in evidence (from moderate to low level) because the sample sizes appeared inadequate, and all the patients recruited were female. Therefore, we consider the evidence to show only modest symptom improvement with PBM for patients with CIPN at 8 weeks. 
Pain Physician: November/December 2018: 21:571-592

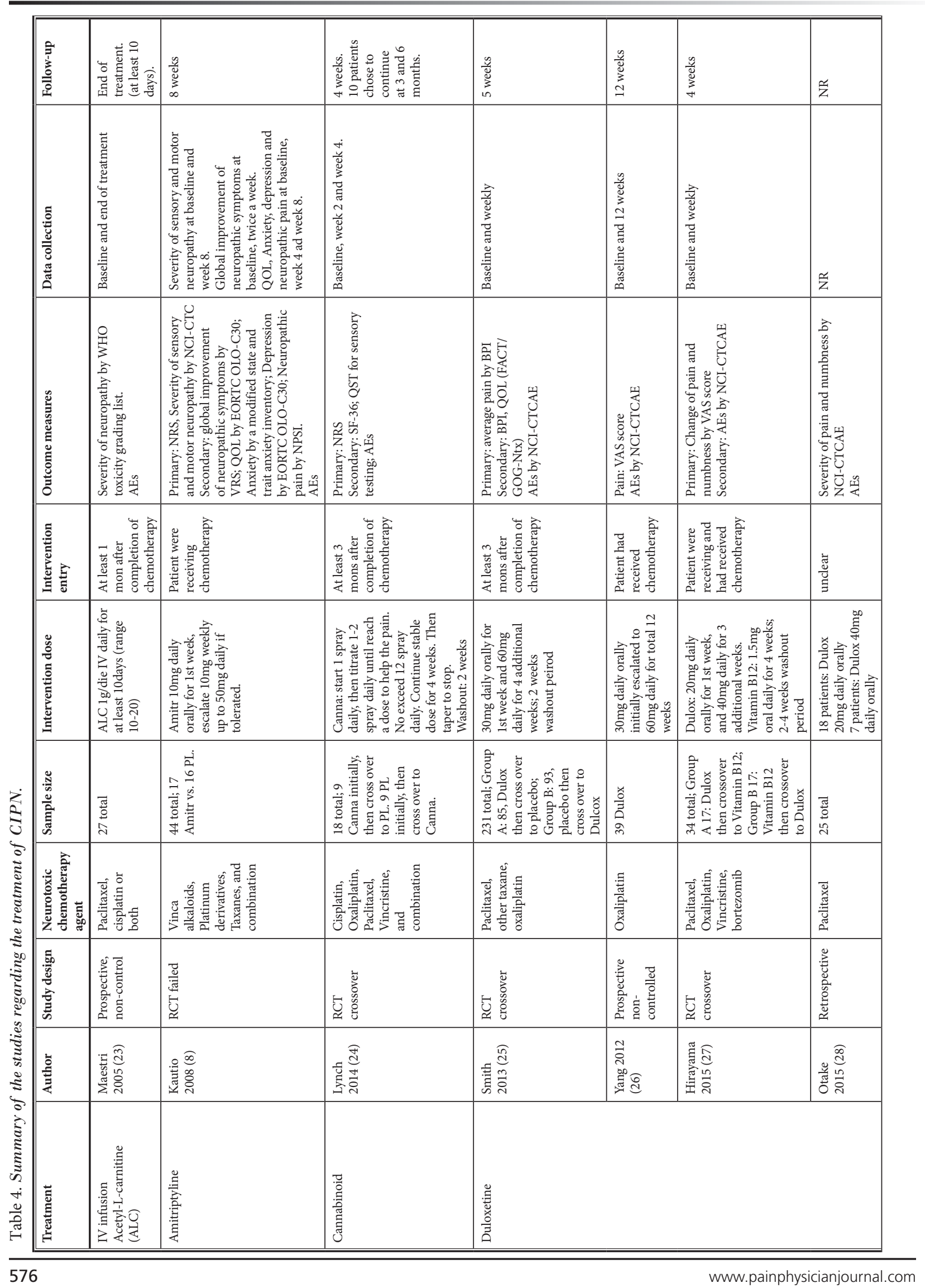


Treatment of Chemotherapy Induced Peripheral Neuropathy

\begin{tabular}{|c|c|c|c|c|c|}
\hline 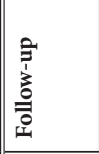 & 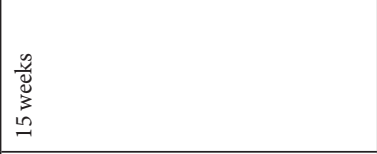 & 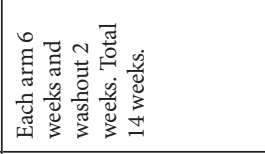 & 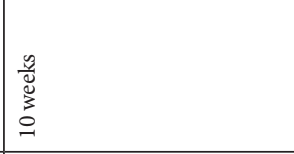 & 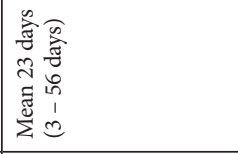 & 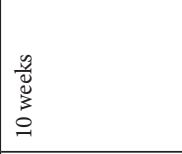 \\
\hline 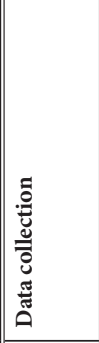 & 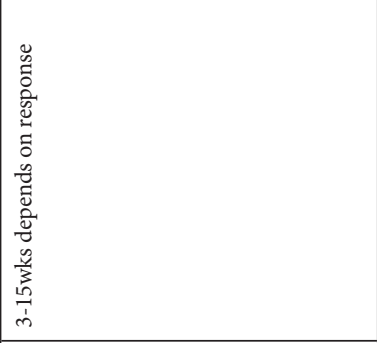 & 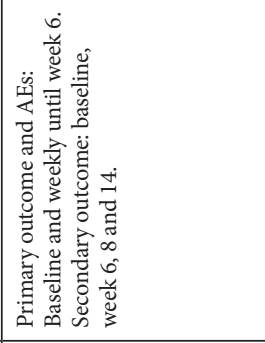 & 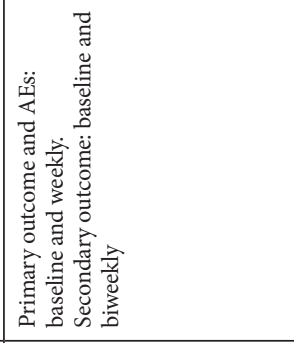 & 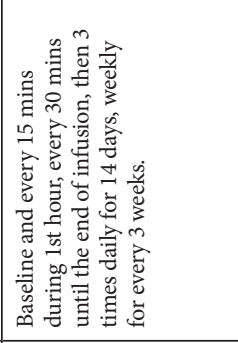 & 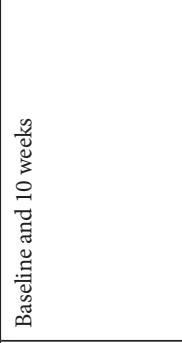 \\
\hline 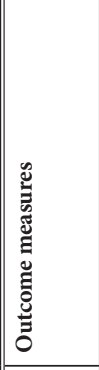 & 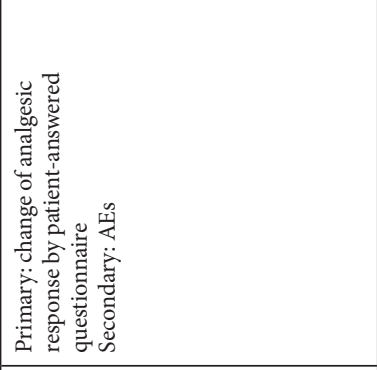 & 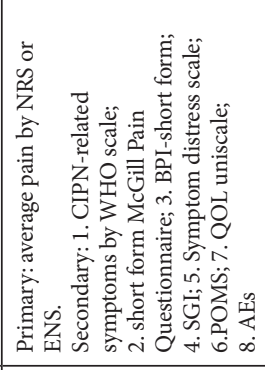 & 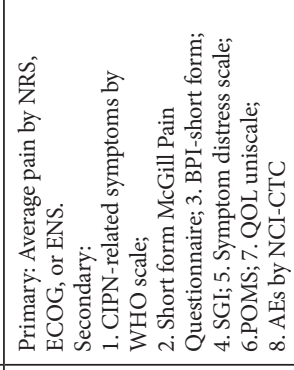 & 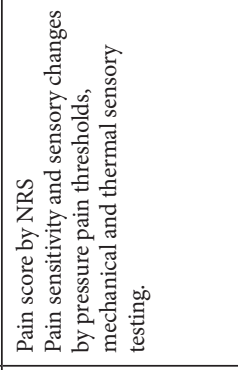 & 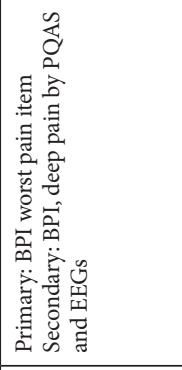 \\
\hline 总 & 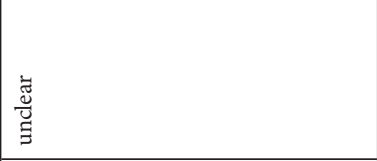 & 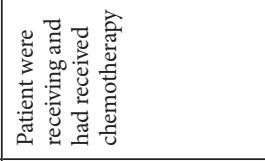 & 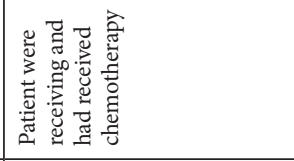 & 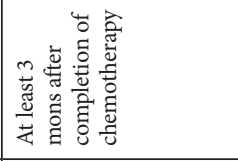 & 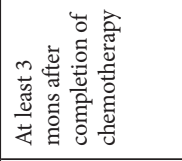 \\
\hline 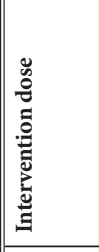 & 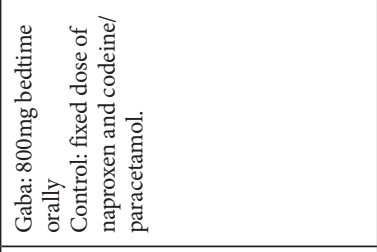 & 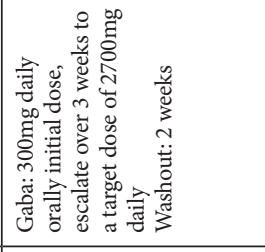 & 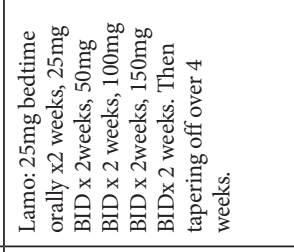 & 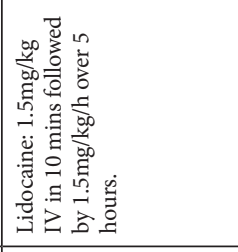 & 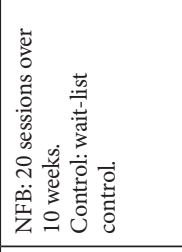 \\
\hline 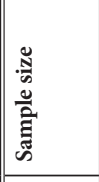 & 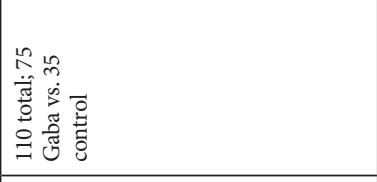 & 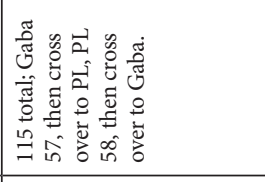 & 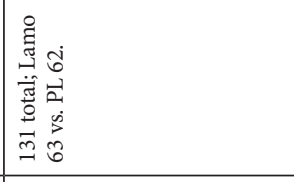 & 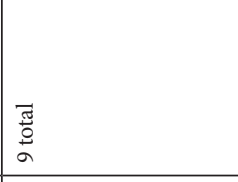 & 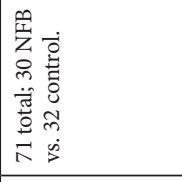 \\
\hline 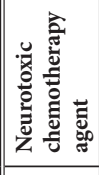 & 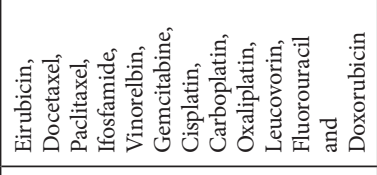 & 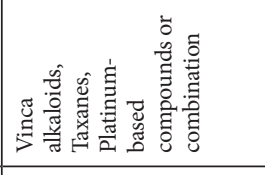 & 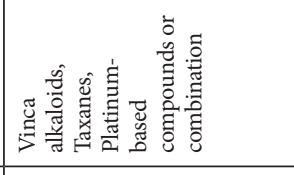 & 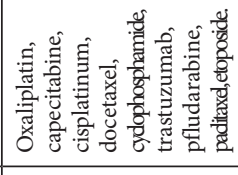 & 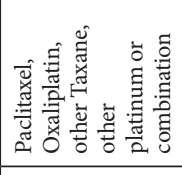 \\
\hline 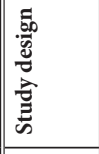 & 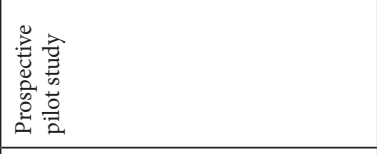 & 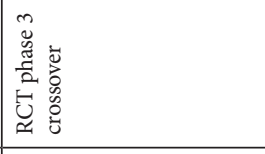 & 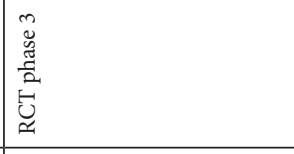 & 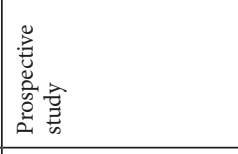 & $\underset{\varkappa}{\dddot{\varkappa}}$ \\
\hline 总 & 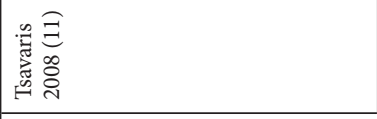 & 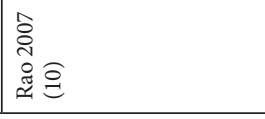 & 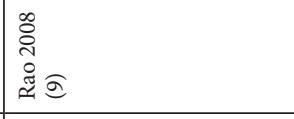 & 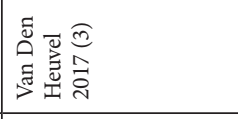 & 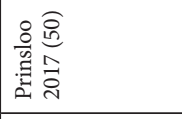 \\
\hline 产 & 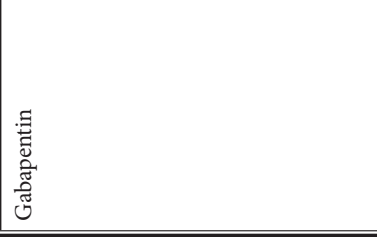 & & 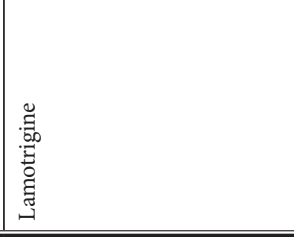 & 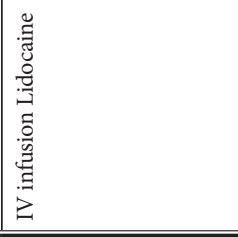 & 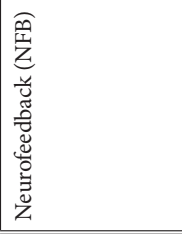 \\
\hline
\end{tabular}


Pain Physician: November/December 2018: 21:571-592

\begin{tabular}{|c|c|c|c|c|c|c|c|c|}
\hline 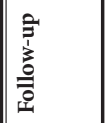 & 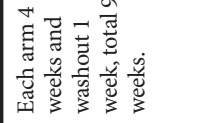 & 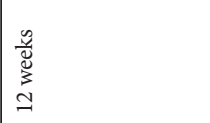 & 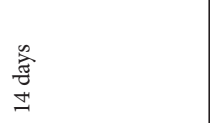 & 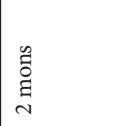 & 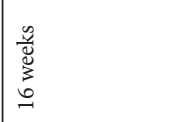 & 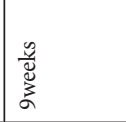 & 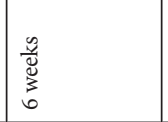 & 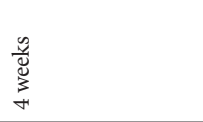 \\
\hline 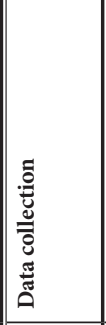 & 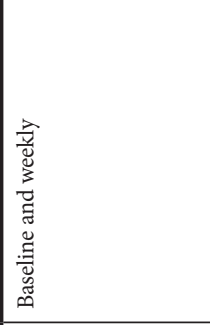 & 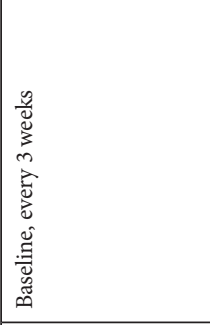 & 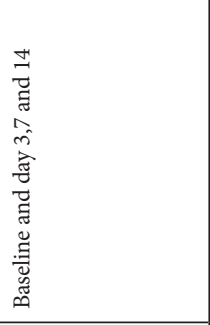 & 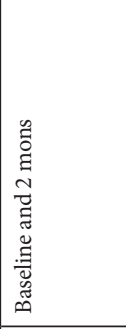 & 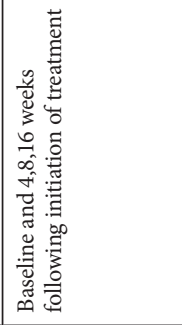 & 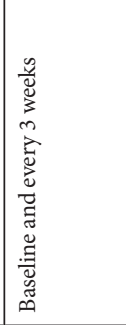 & 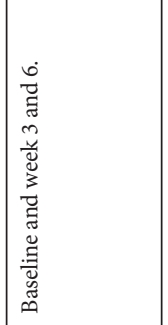 & 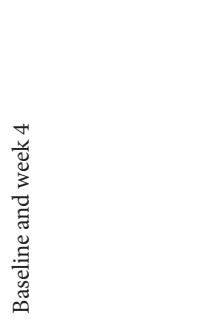 \\
\hline 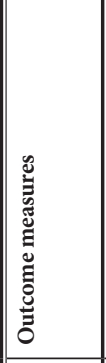 & 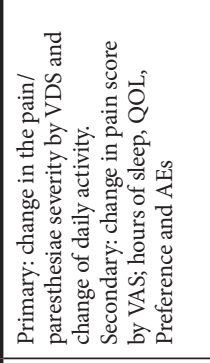 & 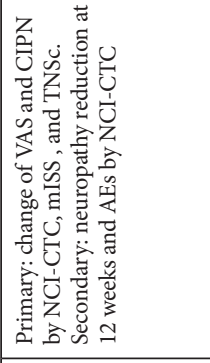 & 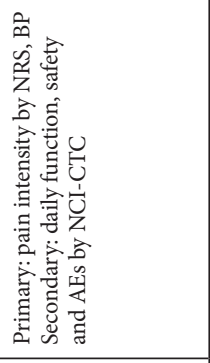 & 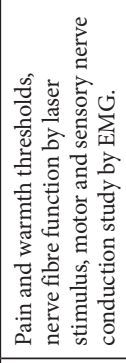 & 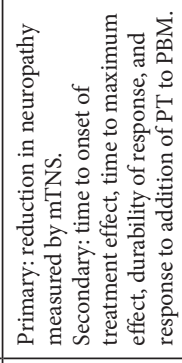 & 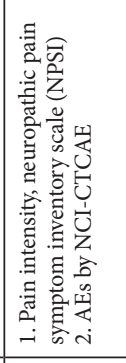 & 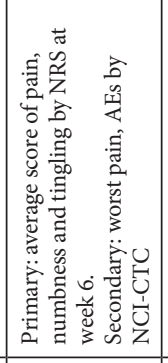 & 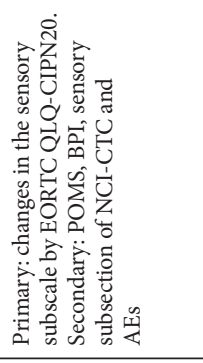 \\
\hline 总 & 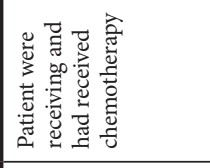 & 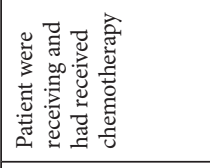 & 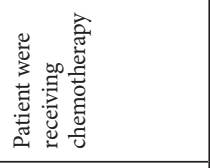 & 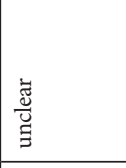 & 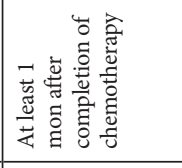 & 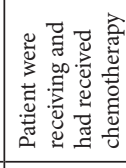 & 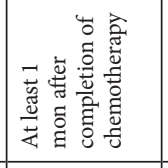 & 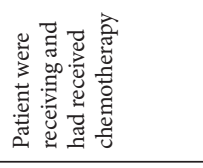 \\
\hline 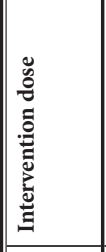 & 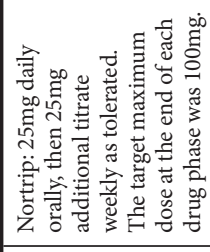 & 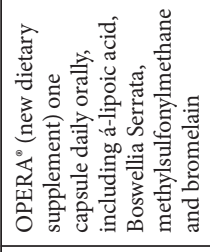 & 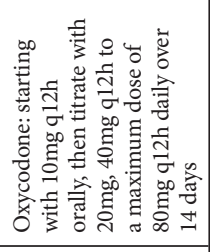 & 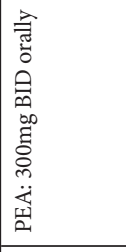 & 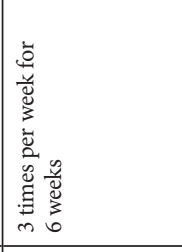 & 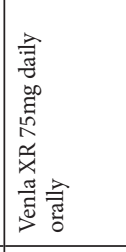 & 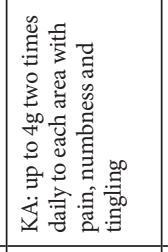 & 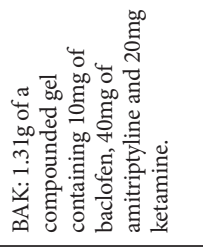 \\
\hline 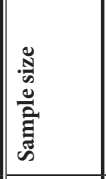 & 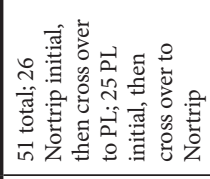 & 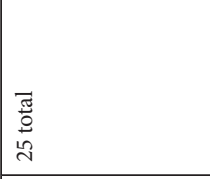 & 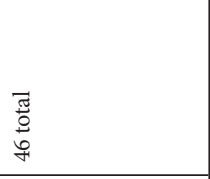 & 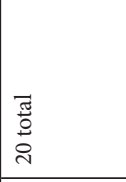 & 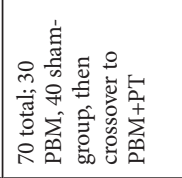 & 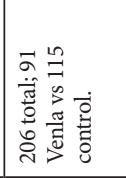 & 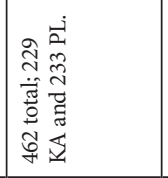 & 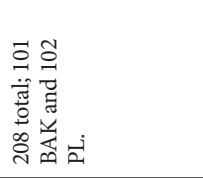 \\
\hline 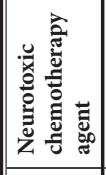 & 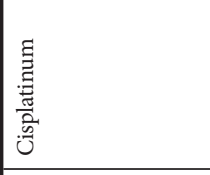 & 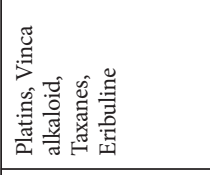 & 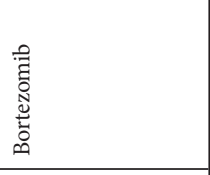 & 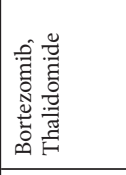 & 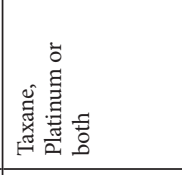 & 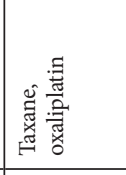 & 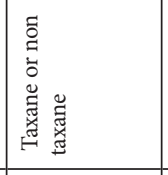 & 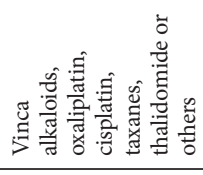 \\
\hline 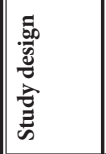 & 悹 & 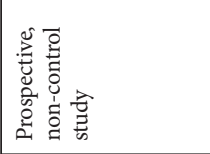 & 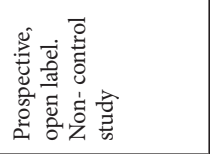 & 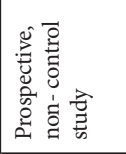 & $\overrightarrow{\mathscr{\varkappa}}$ & 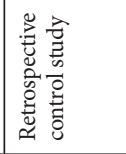 & 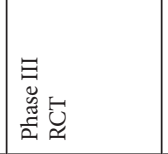 & $\underset{\mathscr{E}}{H}$ \\
\hline $\mid$\begin{tabular}{||l} 
总 \\
\end{tabular} & 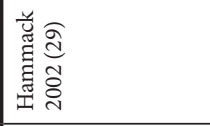 & 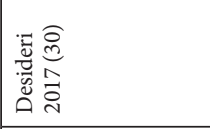 & 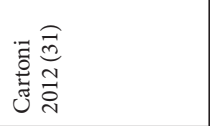 & 春 & 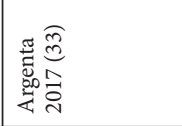 & 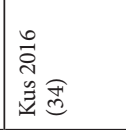 & 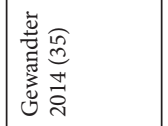 & 惫 \\
\hline 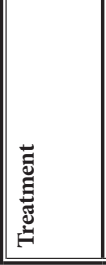 & 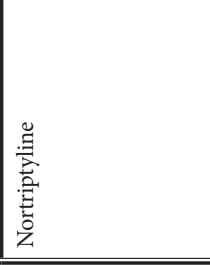 & 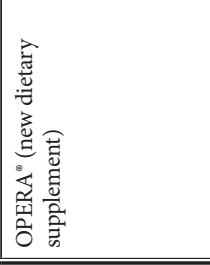 & 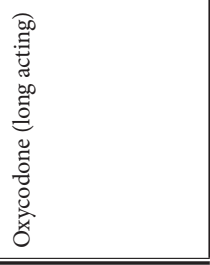 & 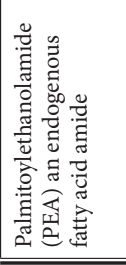 & 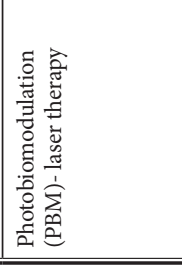 & 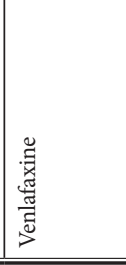 & 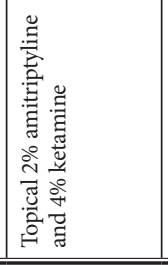 & 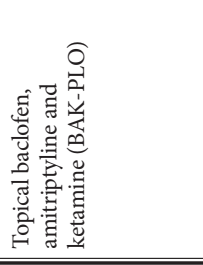 \\
\hline
\end{tabular}


Treatment of Chemotherapy Induced Peripheral Neuropathy

\begin{tabular}{|c|c|c|c|c|c|c|}
\hline 产 & 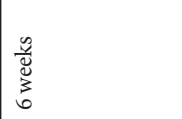 & 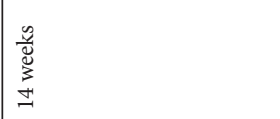 & 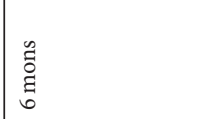 & 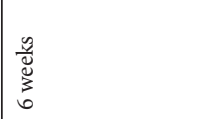 & 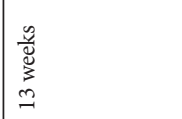 & 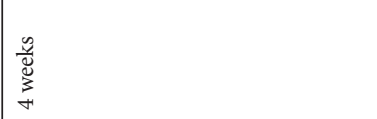 \\
\hline 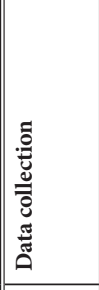 & 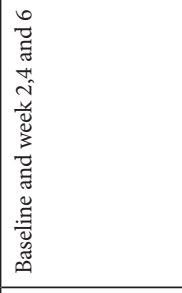 & 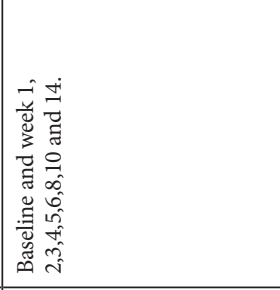 & 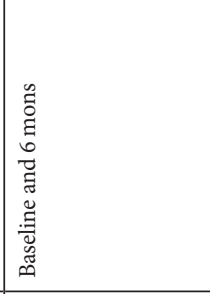 & 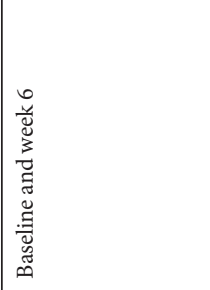 & 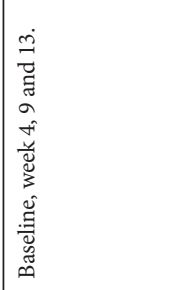 & 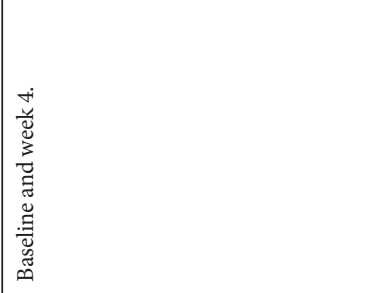 \\
\hline 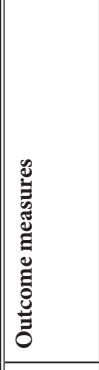 & 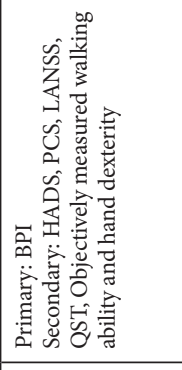 & 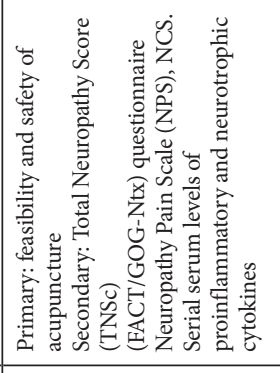 & $\mid \begin{array}{r}\tilde{z} \\
z\end{array}$ & 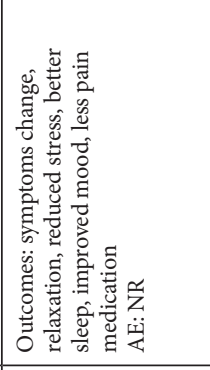 & 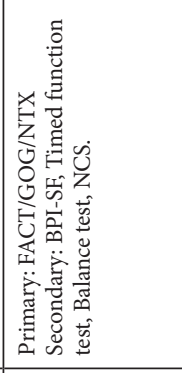 & 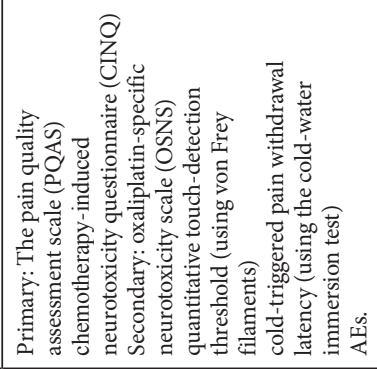 \\
\hline 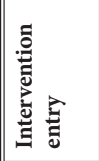 & 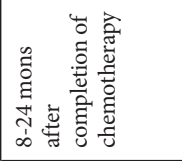 & 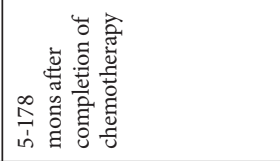 & 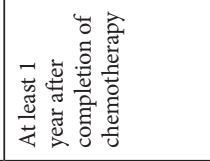 & 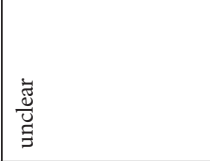 & 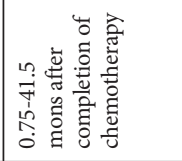 & 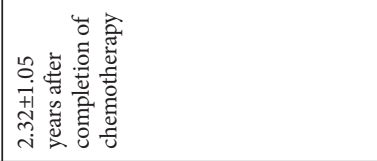 \\
\hline 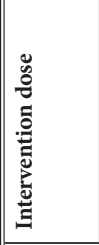 & 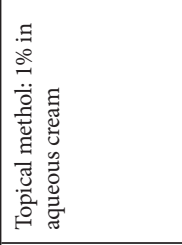 & 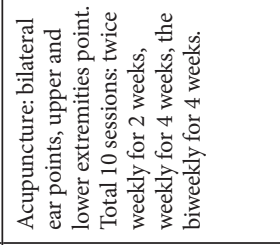 & 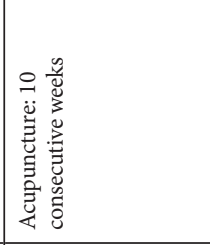 & 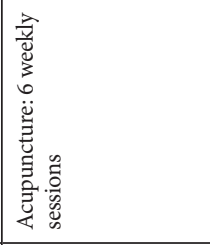 & 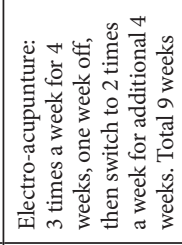 & 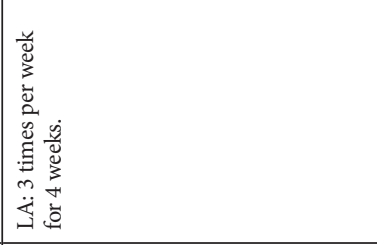 \\
\hline 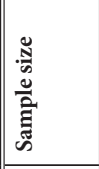 & 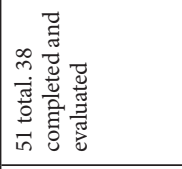 & 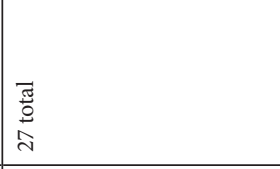 & 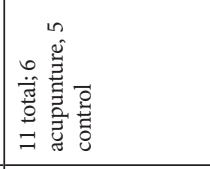 & 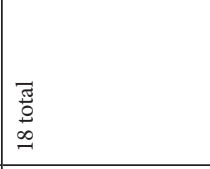 & $\begin{array}{l}\text { 丞 } \\
\stackrel{N}{N}\end{array}$ & 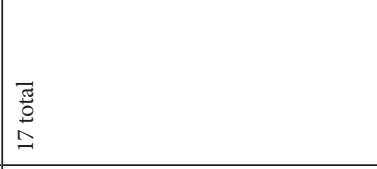 \\
\hline 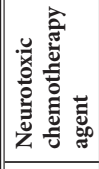 & 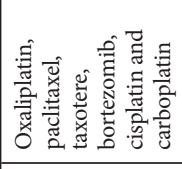 & 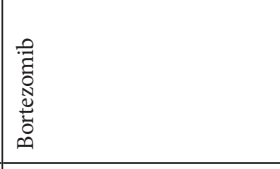 & 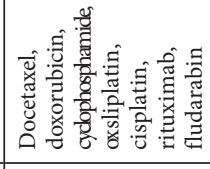 & 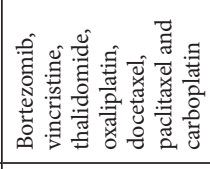 & 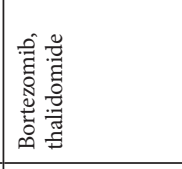 & 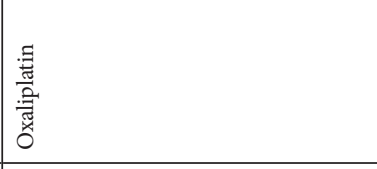 \\
\hline 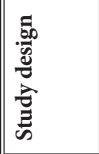 & 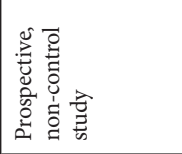 & 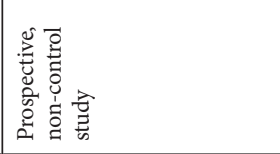 & 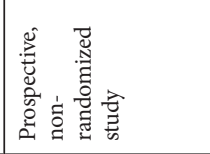 & 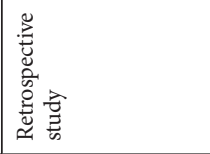 & 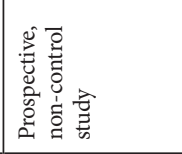 & 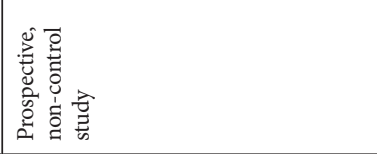 \\
\hline 总 & 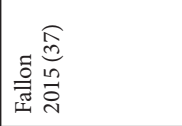 & 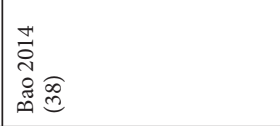 & 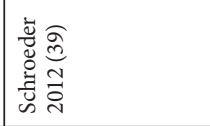 & 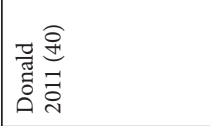 & 递 & 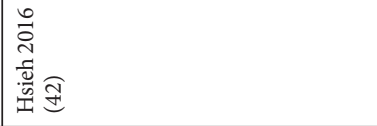 \\
\hline 离 & 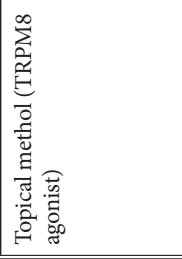 & 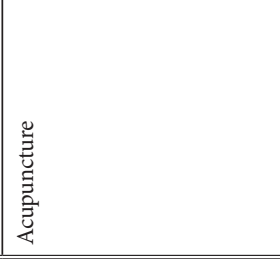 & & & 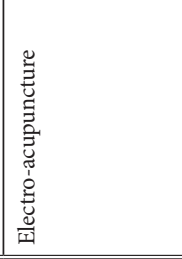 & 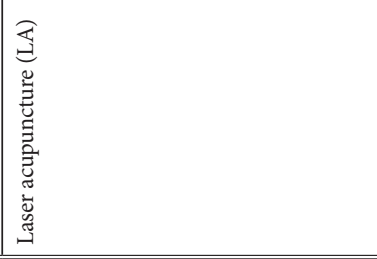 \\
\hline
\end{tabular}


Pain Physician: November/December 2018: 21:571-592

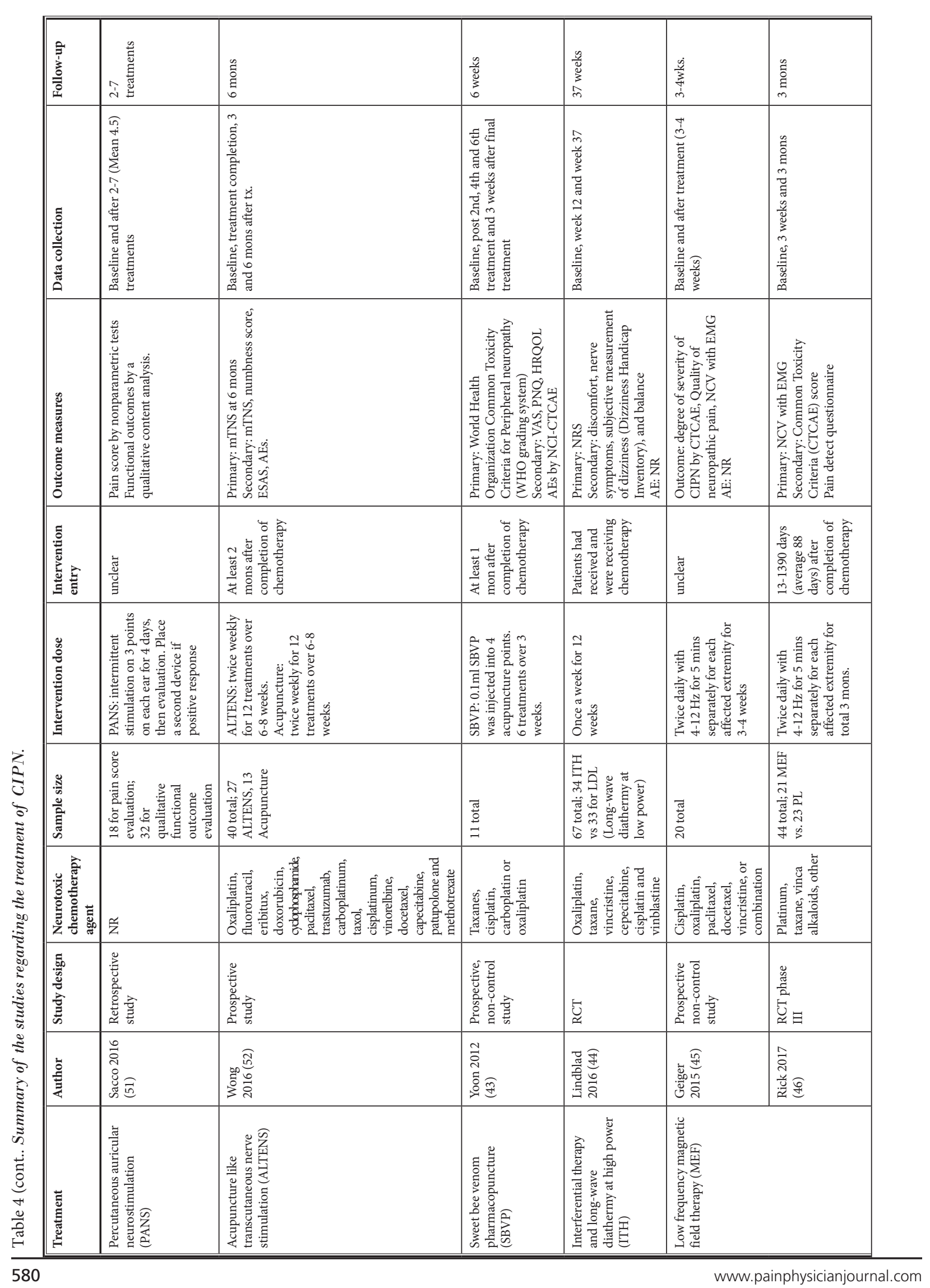


Treatment of Chemotherapy Induced Peripheral Neuropathy
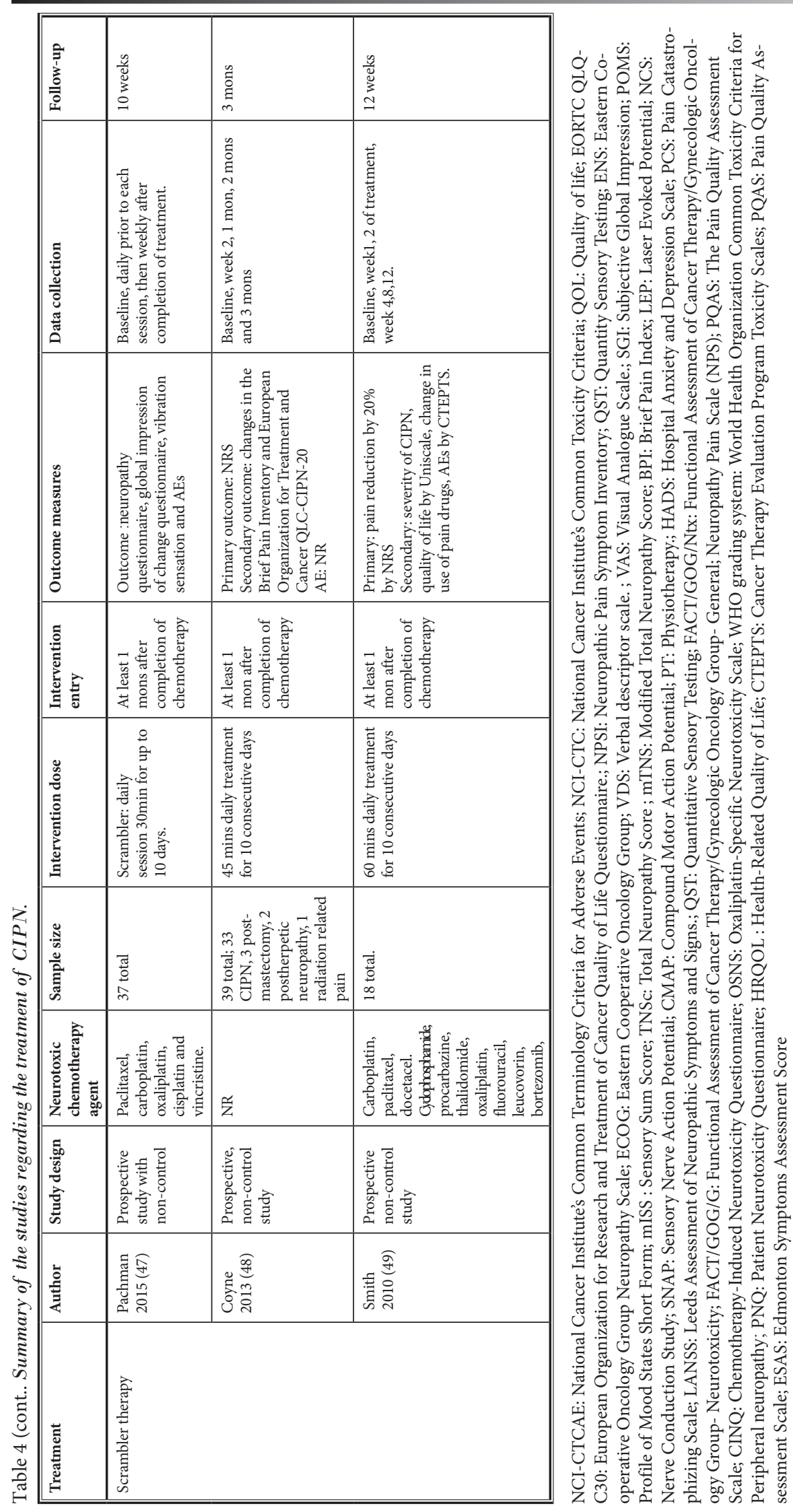

Adverse Events

Of the included studies, 24 examined the AEs associated with treatments for CIPN. Most reported that the AEs were mild and tolerable. No studies were prematurely closed because of high toxicity. Most studies used $\mathrm{NCl}$ Common Terminology Criteria for Adverse Events ( $\mathrm{NCl}-\mathrm{CTCAE})$ or $\mathrm{NCl}$ Common Toxicity Criteria ( $\mathrm{NCl}-$ CTC) to evaluate AEs, but a few studies used the World Health Organization (WHO) Common Toxicity Criteria for peripheral neuropathy toxicity evaluation. Only 2 studies reported $\mathrm{NCl}-\mathrm{CTCAE}$ Grade $3 \mathrm{AEs}$. One is the large RCT crossover study for duloxetine reporting that $16 \%$ of patients in the duloxetine group vs. $27 \%$ in the placebo group had Grade 2 AEs, and that $7 \%$ of patients in duloxetine group vs. $3 \%$ in the placebo group had Grade 3 AEs (25). Another study enrolled 46 patients with CIPN to be treated with oxycodone, 18 of these patients had mild AEs, and only 1 patient had Grade 3 AEs (31).

\section{Discussion}

This systematic review provided up-to-date evidence-based recommendations for the treatment of CIPN. We were unable to make a recommendation for 22 of the 26 examined therapeutic modalities for various reasons, including inconclusive evidence, a limited number of studies, low statistical power, flaws in trial design, and inconsistency 


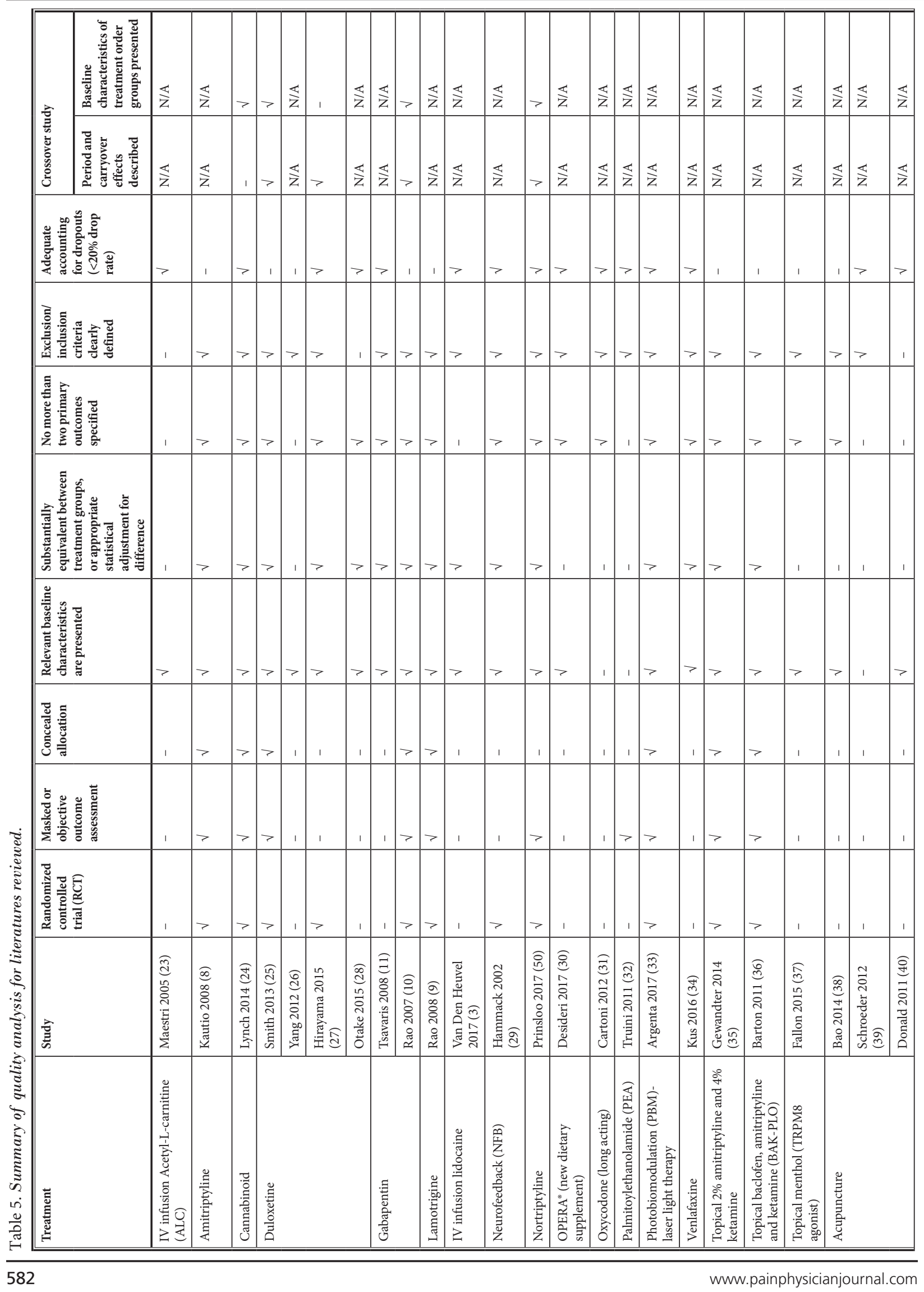




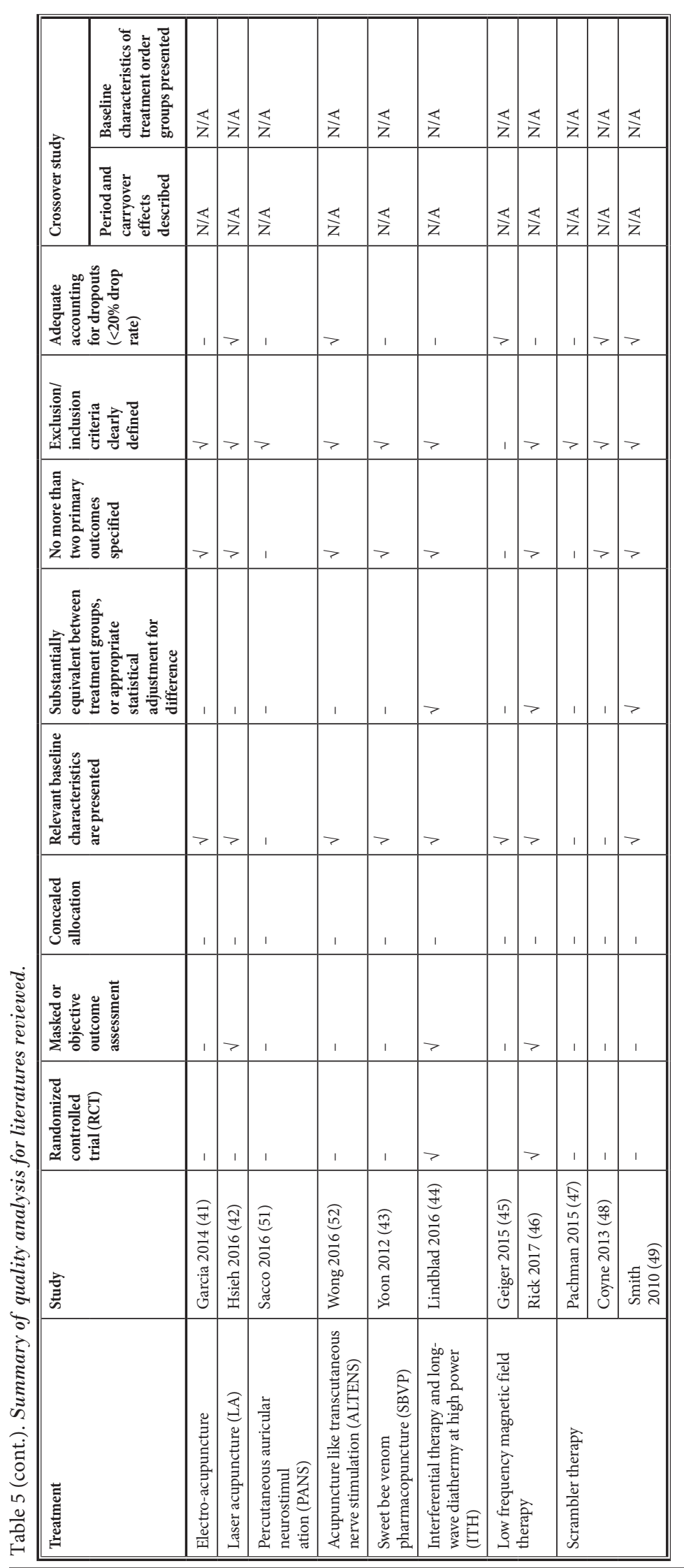

between studies. However, we were able to develop recommendations for 4 of the examined modalities: clinicians may offer duloxetine or PBM, but they should not offer lamotrigine or topical $K A$. To improve the confidence in the results of clinical studies, 3 aspects of study design should be standardized: primary outcome measurement, chemotherapy regimens, and the timing of treatment entry.

\section{Primary Outcome Measurements}

A primary outcome measure was reported in 26 of the total 35 studies. Primary outcome measures included pain intensity by the Numeric Rating Scale (NRS), or Neuropathy Scale (ENS), Eastern Cooperative Oncology Group (ECOG) (18 studies), severity of neuropathy by $\mathrm{NCl}$-CTCAE (4 studies), TNS or mTNS (4 studies), nerve conduction study (NCS) (4 studies), the Brief Pain Inventory (BPI) pain intensity (3 studies), the Neuropathic Pain Symptom Inventory (NPSI) (1 study), and the Pain Quality Assessment Scale (PQAS) (1 study), etc. No consensus exists regarding which of these measures are the best for assessing the outcomes of CIPN treatment. CIPN is a very common side effect of cancer treatment and cancer survivors present with sensory, motor and autonomic neuropathy. Clinically most patients with CIPN have sensory neuropathy, and among these patients, pain is one of the main complaint. However, some patients may have numbness, tingling, and gait imbalance as primary complaints. A few studies reported significant improvement in neuropathy but no change in pain intensity because the enrolled patients presented with mixed symptoms of neuropathy and not all patients had pain. This lack of standardized primary outcome measurements necessitates further studies that characterize and separate CIPN- related pain from other sensory symptoms; using standard outcome measures will help to determine the best outcome measure for 
Pain Physician: November/December 2018: 21:571-592

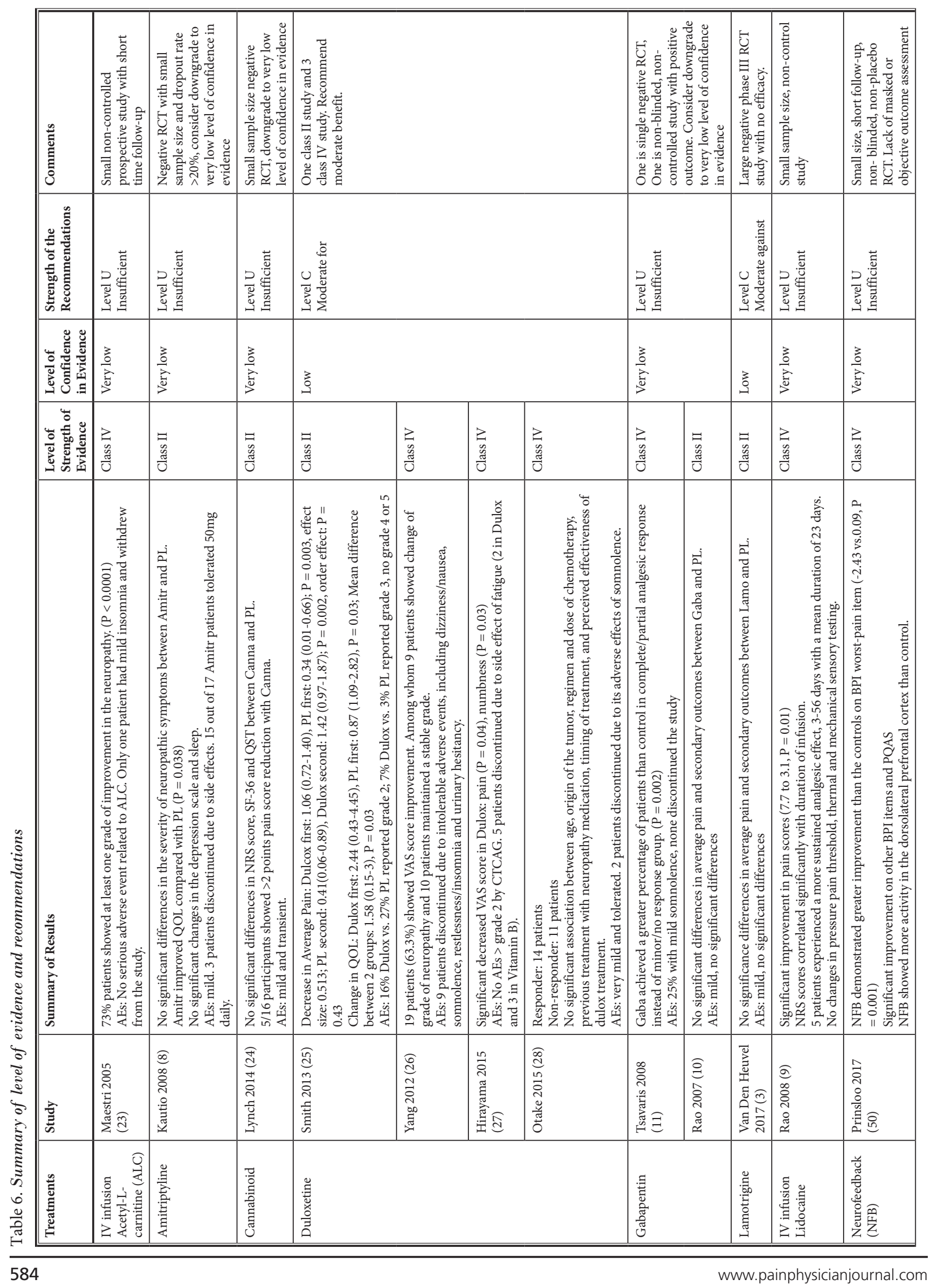


Treatment of Chemotherapy Induced Peripheral Neuropathy

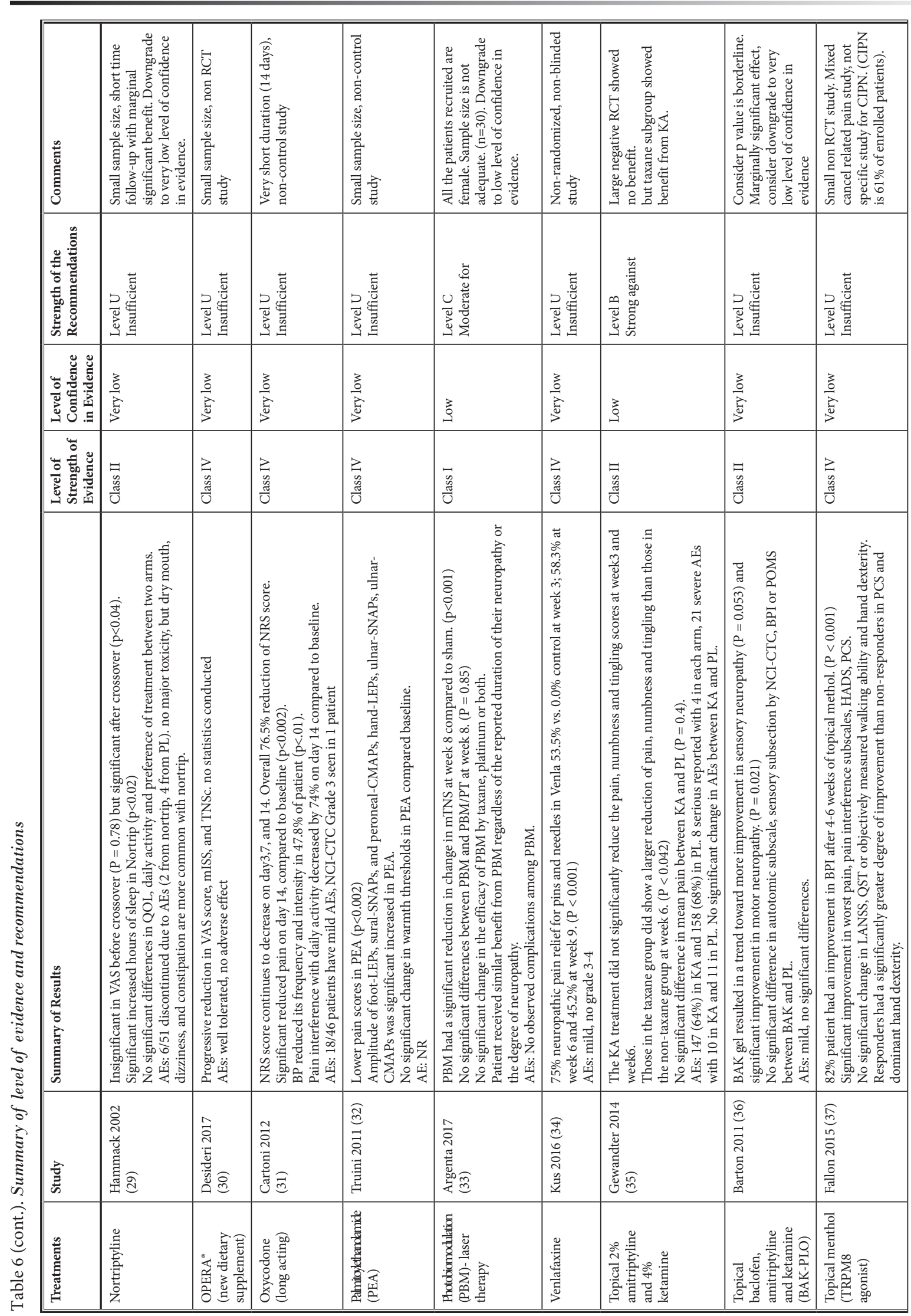


Pain Physician: November/December 2018: 21:571-592

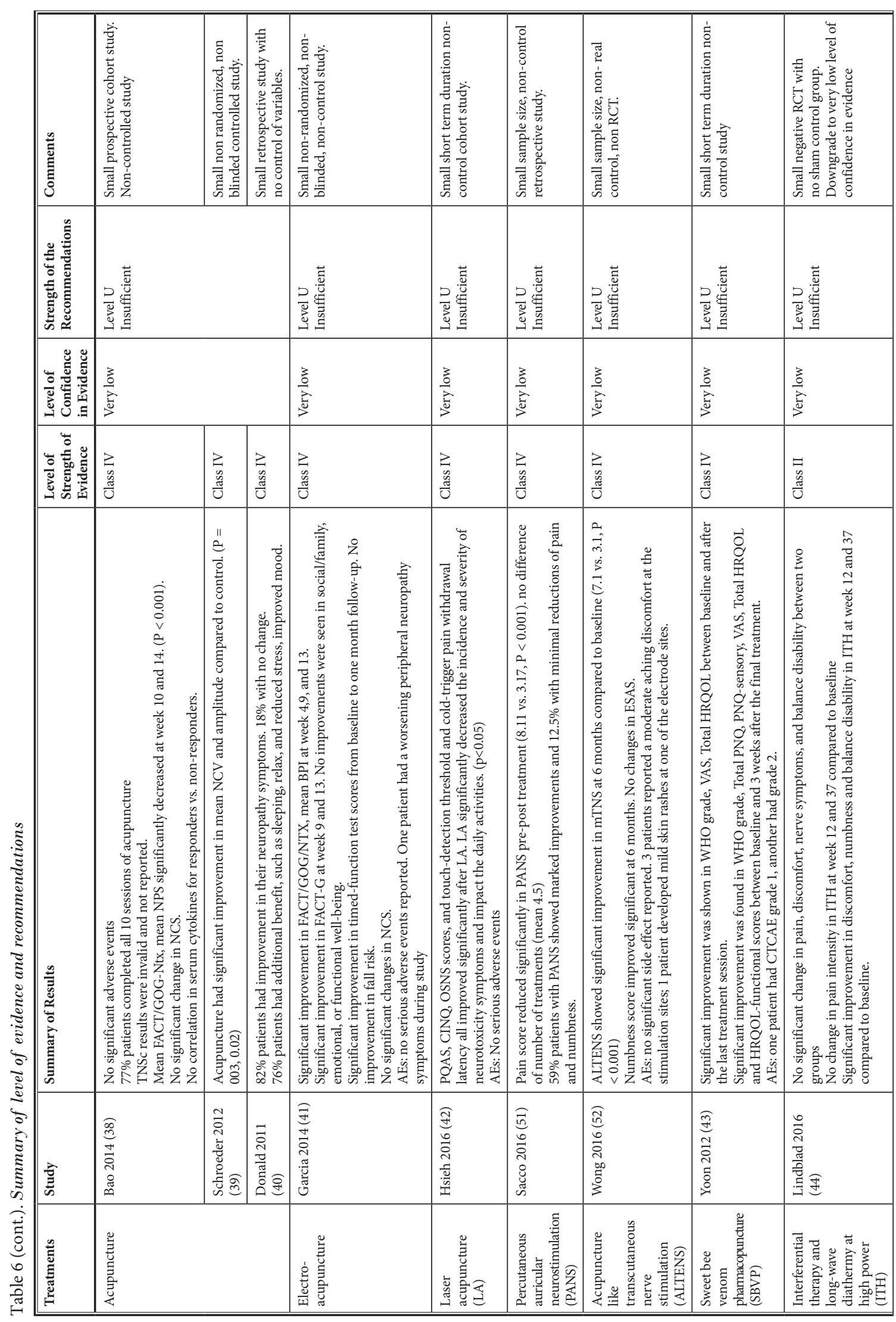




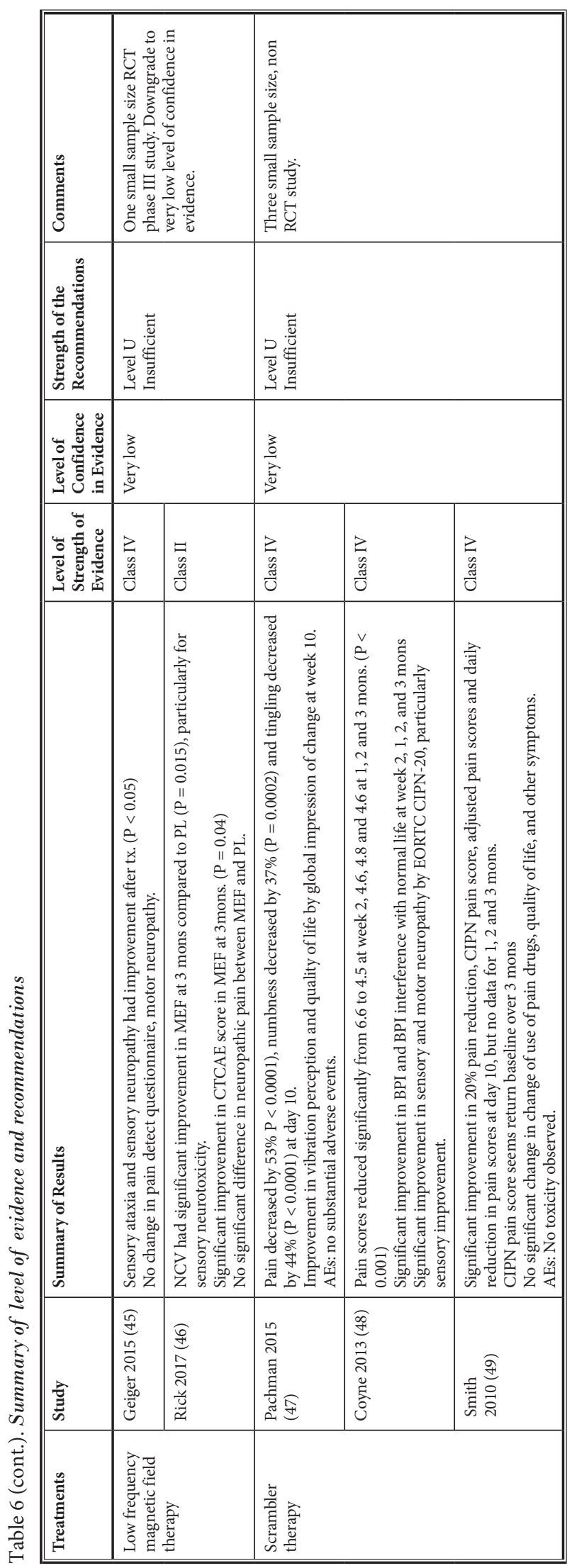

future CIPN treatment trials. Here we suggest that only CIPN patients who have an NRS pain score higher than $4 / 10$ should be enrolled in trials in which pain intensity is primary outcome measure.

As far as the severity of the neuropathy evaluation, there are several commonly used tools including $\mathrm{NCl}-\mathrm{CTCAE}$, TNS/mTNS, WHO toxicity grading, NCS/ EMG, the FACT/GOG-Ntx, EORTC-CIPN20 and PNQ. $\mathrm{NCl}-\mathrm{CTCAE}$ is widely employed owing to its ease of use; it includes 2 items for use in clinical assessment of sensory and motor neuropathy, rated on a 0-4 grading scale. However, this tool is limited by its high rates of inter-observer disagreement and low sensitivity $(8,28,53)$. TNS has 7 items covering 7 domains, sensory, motor, autonomic symptoms, pin sensitivity, vibration sensitivity, strength, and tendon reflexes, whereas modified TNS (mTNS) does not include autonomic symptom. mTNS was the most highly rated clinical assessment tool in a recent Delphi survey, and enables assessment of both small and large nerve fiber function (54). It is consistently reproducible and correlates with TNS, the gold standard of neuropathy assessment $(33,53,55,56)$. The WHO toxicity grading scale, first published in 1979, developed a standardized approach to tumor treatment-related toxicity. The peripheral neuropathy section of this scale included measurements of paranesthesia, changes in deep tendon reflexes, and the extent of motor loss. However, it never became widely used for the assessment of CIPN (53). NCS/EMG is painful, time consuming, and only detects large myelinated fibers of involved peripheral nerves. Because some chemotherapy drugs, for instance bortezomib, affects small myelinated and unmyelinated fibers, these tests are not ideal for assessing CIPN or for monitoring therapeutic response. The FACT/GOG-Ntx and the EORTC-CIPN20 scales have high sensitivity, are directly relevant to CIPN-related functional deficits, and are well correlated with objective measures of neuropathy (57). FACT/GIG-Ntx contains 11 items divided into 5 sections: physical well-being, social/family well-being, emotional well-being, functional well-being, and additional concerns. EORTC-CIPN20 contains 20 items assessing sensory (9 items), motor (8 items), and autonomic symptoms ( 3 items), on a 0-4 grading scale. Even though FACT/GOG-Ntx and EORTC-CIPN20 appear to be the most comprehensive CIPN assessment tools, they are time consuming and of limited usefulness for primary outcome assessment. However, PNQ consists of only 3 items, rated on a $0-4$ grading scale assessing sensory, motor, and function loss. The PNQ is 
convenient, and can be conducted without extensive equipment or training (54).

Despite broad and viable tools of outcome measures for CIPN, further study should focus on a tool with high reliability and reproducibility across examiners to assess CIPN. Here we consider that FACT/GOG-Ntx andEORTC-CIPN20 are more appropriate for secondary outcome measurements, as they are more comprehensive than some other tools, involving assessments of function and quality of life. From among the currently available assessment tools, we support Cavaletti and colleagues' recommendation to use the TNS/mTNS and measurements of pain intensity by NRS as the primary outcome measurements, as these tools allow the most effective descriptions of the type and severity of CIPN (53). In our review, 2 RCTs $(25,33)$ with strong evidence adopted pain intensity and mTNS as primary outcome respectively.

\section{Chemotherapeutic Agents}

In order to better treat CIPN, a better understanding of the pathophysiological mechanisms of CIPN is needed. It is unclear why some agents that benefit diabetic peripheral neuropathic pain are not effective for treating CIPN $(8,10,11)$. Several mechanisms are involved in diabetic peripheral neuropathy; for example, diabetes mellitus causes changes in ion channel expression in peripheral nerve fibers, which in turn leads to hyperexcitability. Therefore, in patients with diabetic neuropathic pain, dysregulation of voltage-gated calcium channels leads to an enhanced calcium influx in sensory neurons (58). The medications pregabalin and gabapentin are approved by the Food and Drug Administration for the first-line treatment of diabetic neuropathic pain because they selectively bind to presynaptic voltage-gated calcium channels in the brain and spinal cord, inhibiting of the release of excitatory neurotransmitters (59-61). However, those medications are not effective in CIPN, suggesting the mechanisms of CIPN differ from those underlying other forms of peripheral neuropathy.

The mechanisms of CIPN also seem to depend upon the type of chemotherapeutic medication used. CIPN can by caused by antimicrotubule agents (taxanes, including paclitaxel and docetaxel), platinum compounds (cisplatin, carboplatin, oxaliplatin), proteasome inhibitors (bortezomib), immunomodulatory agents (thalidomide and lenalidomide) and vinca alkaloids (vincristine, vinblastine, and vinorelbine). Bort- ezomib causes a painful length dependent small fiber axonal sensory neuropathy by inhibiting proteasomes, the primary intracellular protein degradation machinery in neurons (62). It also increases microtubule polymerization, and causes mitochondria to exhibit decreased axonal transport and function in sensory neurons (62). Thalidomide is thought to cause peripheral neuropathy via both its immunomodulation and antiangiogenic effects, resulting in partially irreversible damage to distal axons and dorsal root ganglion (DRG) neurons from capillary damage and secondary anoxemia in nerve fibers $(63,64)$. The platinum-based chemotherapeutics drugs work by forming interstrand DNA adducts, leading to cell cycle arrest, but this antineoplastic mechanism may also cause CIPN through damaging DRG by forming adducts with nuclear and mitochondrial DNA $(65,66)$. In addition, oxaliplatin's effect on voltage-gated sodium channel kinetics causes cold-induced dysesthesia in the hands and mouth (67). Taxane agents prevent microtubule depolymerization by binding to polymerized tubulin within microtubules in sensory neurons (68). Vinca alkaloids, which are used for the primary treatment of hematological malignancies, predominantly cause sensory neuropathy, with some degree of motor involvement. In the contrast to the taxane agents, vinca alkaloids promote microtubule depolymerization by binding to microtubules, disrupting mitotic spindles and causing cell cycle arrest (68). DRG sensory neurons, which are highly polarized, require proper microtubule function for axonal transport of mRNAs, proteins, mitochondria and other organelles. It helps to explain why these 2 drugs with opposite effects on microtubule stability both can cause CIPN $(69,70)$. Although the mechanisms underlying CIPN are varied and unclear, most human and animal studies implicate axonal degeneration as a common process in CIPN pathology, which include defects in axon transport, altered mitochondrial function and altered calcium ion homeostasis (7).

A large negative RCT of topical KA reported no significant improvement in pain, numbness, and tingling scores at 3 and 6 weeks (35). However, the subgroup of patients who underwent taxane chemotherapy had significantly lower pain, numbness, and tingling scores than did patients in the non-taxane group at 6 weeks (35). These results suggest that topical KA may have benefits for patients whose CIPN is attributable to taxane agents. Given the varied mechanisms of chemotherapeutic agents, we consider that future study 
designs should focus on a single type of chemotherapeutic agent causing CIPN in order to better determine the efficacy of CIPN treatment.

\section{Timing of Treatment Entry}

Table 4 outlines the timing of treatment entry for each study. Nineteen (54\%) studies reported that CIPN treatment was initiated after completion of chemotherapy. Of those 19 studies, only 9 reported that the treatment started at least 3 months after completion of chemotherapy. In addition, 2 (6\%) studies reported that the treatment started while patients were undergoing chemotherapy. Eight (23\%) studies reported the timing of treatment occurred while patients had received and were receiving chemotherapy. Six $(17 \%)$ studies were unclear on the timing of chemotherapy.

CIPN is fairly common within 1 month after cessation of chemotherapy, and its prevalence decreases over time. Approximately one-third of patients have chronic CIPN lasting 6 months or more after the end of chemotherapy (1). The lack of uniformity in the timing of treatment entry among studies makes the results less convinced and also makes between-study comparisons difficult. In 2 large positive RCTs, the timing of treatment entry was at least 1 or 3 months, respectively, after completion of chemotherapy $(25,33)$. Another 2 large negative RCTs reported that CIPN treatment started while patients had received and were receiving chemotherapy $(9,10)$. These data suggest that the timing of treatment entry should be a standardized element of study design. We consider that at least 3 months after completion of chemotherapy is an appropriate timing of the treatment entry point, as this period would allow adequate time for self-recovery of involved peripheral nerves after cessation of chemotherapy.

\section{Limitations}

This review, while comprehensive, had some limitations. It included only literature published in English and the included studies were relatively poor homogeneous, with variations in timing of treatment, primary outcomes and chemotherapeutic agents used.

\section{Conclusions}

These evidence-based clinical recommendations aimed to provide the best and most current evidence on the treatment of CIPN for physicians and patients. They have potential to improve the appropriateness and effectiveness of patient care by employing the best evidence available. In addition, these recommendations serve to identify knowledge, study design, and methodology gaps in the clinical literature on the treatment of CIPN. Future study should focus on using a standardized study design and outcome measures, large sample sizes and long-term follow-up for CIPN treatment trials.

\section{Acknowledgments}

Author Contributions: All authors had full access to all the data in the study and takes responsibility for the integrity of the data and the accuracy of the data analysis. Drs. Hou and Abdi designed the study protocol. Drs. Hou, Huh and Abdi managed the literature searches and summaries of previous related work and wrote the first draft of the manuscript. Drs. Huh and Kim provided revision for intellectual content and final approval of the manuscript.

Conflict of Interest: All authors have no conflicts of interest to report. All of the authors of the manuscript did not receive any remuneration.

Funding/support: The study was sponsored by grants from Helen Buchanan and Stanley Joseph Seeger Endowment at The University of Texas MD Anderson Cancer Center to Dr. Abdi. But the manuscript is separate and apart from the guidance of the sponsor.

Role of Sponsor: The financial sponsor of this work had no role in the design and conduct of the study or the collection, management, analysis, and interpretation of the data. The sponsor also did not have a role in the preparation or review of the manuscript or the decision to submit.

The authors also wish to thank Amy Ninetto, an editor in the Department of Scientific Publications, The University of Texas MD Anderson Cancer Center who edited the manuscript for non-intellectual content. 


\section{REFERENCES}

1. Seretny M, Currie GL, Sena ES, Ramnarine S, Grant R, MacLeod MR, Colvin LA, Fallon M. Incidence, prevalence, and predictors of chemotherapy-induced peripheral neuropathy: A systematic review and meta-analysis. Pain 2014; 155:2461-2470.

2. Miltenburg NC, Boogerd W. Chemotherapy-induced neuropathy: A comprehensive survey. Cancer Treat Rev 2014; 40:872-882.

3. van den Heuvel SAS, van der Wal SEI, Smedes LA, Radema SA, van Alfen N, Vissers KCP. Intravenous lidocaine: Oldschool drug, new purpose-reduction of intractable pain in patients with chemotherapy induced peripheral neuropathy. Pain Res Manage 2017; 2017:8053474.

4. Loprinzi CL, Reeves BN, Dakhil SR, Sloan JA, Wolf SL, Burger KN, Kamal A, Le-Lindqwister NA, Soori GS, Jaslowski A), Novotny PJ, Lachance DH. Natural history of paclitaxel-associated acute pain syndrome: Prospective cohort study NCCTG No8C1. J Clin Oncol 2011; 29:1472-1478.

5. Beijers A, Mols F, Dercksen W, Driessen C, Vreugdenhil G. Chemotherapyinduced peripheral neuropathy and impact on quality of life 6 months after treatment with chemotherapy. Journal of Community and Supportive Oncology 2014; 12:401-406.

6. Briani C, Argyriou AA, Izquierdo C, Velasco R, Campagnolo M, Alberti P, Frigeni B, Cacciavillani M, Bergamo F, Cortinovis D, Cazzaniga M, Bruna J, Cavaletti G, Kalofonos HP. Long-term course of oxaliplatin-induced polyneuropathy: A prospective 2-year follow-up study. Journal of the Peripheral Nervous System 2014; 19:299-306.

7. Fukuda Y, Li Y, Segal RA. A mechanistic understanding of axon degeneration in chemotherapy-induced peripheral neuropathy. Front Neurosci 2017; 11:481.

8. Kautio AL, Haanpaa M, Saarto T, Kalso E. Amitriptyline in the treatment of chemotherapy-induced neuropathic symptoms. J Pain Symptom Manage 2008; 35: 31-39.

9. Rao RD, Flynn PJ, Sloan JA, Wong GY, Novotny $P$, Johnson DB, Gross HM, Renno SI, Nashawaty M, Loprinzi CL. Efficacy of lamotrigine in the management of chemotherapy-induced peripheral neuropathy: A phase 3 randomized, double-blind, placebo-controlled trial, $\mathrm{NolC}_{3}$. Cancer 2008; 112:2802-2808.

10. Rao RD, Michalak JC, Sloan JA, Loprinzi CL, Soori GS, Nikcevich DA, Warner DO, Novotny P, Kutteh LA, Wong GY. Efficacy of gabapentin in the management of chemotherapy-induced peripheral neuropathy: A phase 3 randomized, doubleblind, placebo-controlled, crossover trial (NooC3). Cancer 2007; 110:2110-2118.

11. Tsavaris N, Kopterides P, Kosmas C Efthymiou A, Skopelitis H, Dimitrakopoulos A, Pagouni E, Pikazis D, Zis PV, Koufos C. Gabapentin monotherapy for the treatment of chemotherapy-induced neuropathic pain: A pilot study. Pain Med 2008; 9:1209-1216.

12. Hershman DL, Lacchetti C, Dworkin RH, Lavoie Smith EM, Bleeker J, Cavaletti G, Chauhan C, Gavin P, Lavino A, Lustberg MB, Paice J, Schneider B, Smith ML, Smith T, Terstriep S, Wagner-Johnston N, Bak K, Loprinzi CL, American Society of Clinical O. Prevention and management of chemotherapy-induced peripheral neuropathy in survivors of adult cancers: American Society of Clinical Oncology clinical practice guideline. J Clin Oncol 2014; 32:1941-1967.

13. Staff NP, Grisold A, Grisold W, Windebank AJ. Chemotherapy-induced peripheral neuropathy: A current review. Ann Neurol 2017; 81:772-781.

14. Gewandter JS, Freeman R, Kitt RA, Cavaletti G, Gauthier LR, McDermott MP, Mohile NA, Mohlie SG, Smith AG, Tejani MA, Turk DC, Dworkin RH. Chemotherapy-induced peripheral neuropathy clinical trials: Review and recommendations. Neurology 2017; 89: 859-869.

15. Piccolo J, Kolesar JM. Prevention and treatment of chemotherapy-induced peripheral neuropathy. American Journal of Health-System Pharmacy 2014; 71:19-25.

16. Gronseth GW, LM.; Getchius, TS. Clinical practice guideline process manual. 2011 Ed. 2011.

17. Gronseth GW, LM.; Getchius, TS. Amendments to 2011 American Academy of Neurology Clinical Practice Guideline Process Manual. 2015.

18. Bril V, England J, Franklin GM, Backonja M, Cohen J, Del Toro D, Feldman E, Iverson DJ, Perkins B, Russell JW, Zochodne D. Evidence-based guideline: Treatment of painful diabetic neuropathy: Report of the American Academy of Neurology, the American Association of Neuromuscular and Electrodiagnostic Medicine, and the American Academy of Physical Medicine and Rehabilitation. Neurology 2011; 76:1758-1765.

19. Simpson DM, Hallett $M$, Ashman EJ, Comella CL, Green MW, Gronseth GS, Armstrong MJ, Gloss D, Potrebic S, Jankovic J, Karp BP, Naumann M, So YT, Yablon SA. Practice guideline update summary: Botulinum neurotoxin for the treatment of blepharospasm, cervical dystonia, adult spasticity, and headache: Report of the Guideline Development Subcommittee of the American Academy of Neurology. Neurology 2016; 86:1818-1826.

20. Liberati A, Altman DG, TetzlaffJ, Mulrow C, Gotzsche PC, loannidis JP, Clarke M, Devereaux PJ, Kleijnen J, Moher D. The PRISMA statement for reporting systematic reviews and meta-analyses of studies that evaluate health care interventions: Explanation and elaboration. J Clin Epidemiol 2009; 62:e1-e34.

21. Hou S, Kemp K, Grabois M. A systematic evaluation of burst spinal cord stimulation for chronic back and limb pain. Neuromodulation 2016; 19:398-405.

22. Schellinger PD, Bryan RN, Caplan LR, Detre JA, Edelman RR, Jaigobin C, Kidwell CS, Mohr JP, Sloan M, Sorensen AG, Warach S. Evidence-based guideline: The role of diffusion and perfusion MRI for the diagnosis of acute ischemic stroke: Report of the Therapeutics and Technology Assessment Subcommittee of the American Academy of Neurology. Neurology 2010; 75:177-185.

23. Maestri A, De Pasquale Ceratti A, Cundari S, Zanna C, Cortesi E, Crino L. A pilot study on the effect of acetylL-carnitine in paclitaxel- and cisplatininduced peripheral neuropathy. Tumori 2005; 91:135-138.

24. Lynch ME, Cesar-Rittenberg P, Hohmann AG. A double-blind, placebo-controlled, crossover pilot trial with extension using an oral mucosal cannabinoid extract for treatment of chemotherapyinduced neuropathic pain. J Pain Symptom Manage 2014; 47:166-173.

25. Smith EM, Pang $H$, Cirrincione C, Fleishman S, Paskett ED, Ahles T, Bressler LR, Fadul CE, Knox C, LeLindqwister N, Gilman PB, Shapiro CL. 
Effect of duloxetine on pain, function, and quality of life among patients with chemotherapy-induced painful peripheral neuropathy: A randomized clinical trial. JAMA 2013; 309:1359-1367.

26. Yang YH, Lin JK, Chen WS, Lin TC, Yang SH, Jiang JK, Chang SC, Lan YT, Lin CC, Yen CC, Tzeng CH, Wang WS, Chiang $\mathrm{HL}$, Teng CJ, Teng HW. Duloxetine improves oxaliplatin-induced neuropathy in patients with colorectal cancer: An open-label pilot study. Support Care Cancer 2012; 20:1491-1497.

27. Hirayama $Y$, Ishitani $K$, Sato $Y$, Iyama S, Takada K, Murase K, Kuroda $\mathrm{H}$, Nagamachi Y, Konuma Y, Fujimi A, Sagawa T, Ono K, Horiguchi H, Terui T, Koike K, Kusakabe T, Sato T, Takimoto R, Kobune M, Kato J. Effect of duloxetine in Japanese patients with chemotherapyinduced peripheral neuropathy: A pilot randomized trial. Int J Clin Oncol 2015; 20:866-871.

28. Otake A, Yoshino K, Ueda Y, Sawada K, Mabuchi S, Kimura T, Kobayashi E, Isobe A, Egawa-Takata T, Matsuzaki S, Fujita M, Kimura T. Usefulness of duloxetine for Paclitaxel-induced peripheral neuropathy treatment in gynecological cancer patients. Anticancer Res 2015; 35:359-363.

29. Hammack JE, Michalak JC, Loprinzi CL, Sloan JA, Novotny PJ, Soori GS, Tirona MT, Rowland KM, Jr., Stella PJ, Johnson JA. Phase III evaluation of nortriptyline for alleviation of symptoms of cis-platinum-induced peripheral neuropathy. Pain 2002; 98:195-203.

30. Desideri I, Francolini G, Becherini C, Terziani F, Delli Paoli C, Olmetto E, Loi M, Perna M, Meattini I, Scotti V, Greto D, Bonomo P, Sulprizio S, Livi L. Use of an alpha lipoic, methylsulfonylmethane and bromelain dietary supplement (Opera(R)) for chemotherapy-induced peripheral neuropathy management, a prospective study. Med Oncol 2017; 34:46.

31. Cartoni C, Brunetti GA, Federico V, Efficace F, Grammatico S, Tendas A, Scaramucci L, Cupelli L, D’Elia GM, Truini A, Niscola P, Petrucci MT. Controlledrelease oxycodone for the treatment of bortezomib-induced neuropathic pain in patients with multiple myeloma. Support Care Cancer 2012; 20:2621-2626.

32. Truini A, Biasiotta A, Di Stefano G, La Cesa S, Leone C, Cartoni C, Federico V, Petrucci MT, Cruccu G. Palmitoylethanolamide restores myelinated-fibre function in patients with chemotherapy- induced painful neuropathy. CNS Neurol Disord Drug Targets 2011; 10:916-920.

33. Argenta PA, Ballman KV, Geller MA, Carson LF, Ghebre R, Mullany SA, Teoh DG, Winterhoff BJ, Rivard CL, Erickson BK. The effect of photobiomodulation on chemotherapy-induced peripheral neuropathy: A randomized, sham-controlled clinical trial. Gynecol Oncol 2017; 144:159-166.

34. Kus T, Aktas G, Alpak G, Kalender ME, Sevinc A, Kul S, Temizer M, Camci C. Efficacy of venlafaxine for the relief of taxane and oxaliplatin-induced acute neurotoxicity: A single-center retrospective case-control study. Support Care Cancer 2016; 24:2085-2091.

35. Gewandter JS, Mohile SG, Heckler CE, Ryan JL, Kirshner JJ, Flynn PJ, Hopkins JO, Morrow GR. A phase III randomized, placebo-controlled study of topical amitriptyline and ketamine for chemotherapy-induced peripheral neuropathy (CIPN): A University of Rochester CCOP study of 462 cancer survivors. Support Care Cancer 2014; 22:1807-1814.

36. Barton DL, Wos EJ, Qin R, Mattar BI, Green NB, Lanier KS, Bearden JD, 3rd, Kugler JW, Hoff KL, Reddy PS, Rowland KM, Jr., Riepl M, Christensen B, Loprinzi CL. A double-blind, placebocontrolled trial of a topical treatment for chemotherapy-induced peripheral neuropathy: NCCTG trial No6CA. Support Care Cancer 2011; 19:833-841.

37. Fallon MT, Storey DJ, Krishan A, Weir CJ, Mitchell R, Fleetwood-Walker SM, Scott AC, Colvin LA. Cancer treatment-related neuropathic pain: proof of concept study with menthol--a TRPM8 agonist. Support Care Cancer 2015; 23:2769-2777.

38. Bao T, Goloubeva O, Pelser C, Porter N, Primrose J, Hester L, Sadowska M, Lapidus R, Medeiros M, Lao L, Dorsey SG, Badros AZ. A pilot study of acupuncture in treating bortezomib-induced peripheral neuropathy in patients with multiple myeloma. Integr Cancer Ther 2014; 13:396-404.

39. Schroeder S, Meyer-Hamme G, Epplee S. Acupuncture for chemotherapy-induced peripheral neuropathy (CIPN): A pilot study using neurography. Acupunct Med 2012; 30:4-7.

40. Donald GK, Tobin I, Stringer J. Evaluation of acupuncture in the management of chemotherapy-induced peripheral neuropathy. Acupunct Med 2011; 29:230-233.

41. Garcia MK, Cohen L, Guo Y, Zhou Y,
You B, Chiang J, Orlowski RZ, Weber D, Shah J, Alexanian R, Thomas S, Romaguera J, Zhang L, Badillo M, Chen Y, Wei Q, Lee R, Delasalle K, Green V, Wang M. Electroacupuncture for thalidomide/ bortezomib-induced peripheral neuropathy in multiple myeloma: A feasibility study. J Hematol Oncol 2014; 7: 41.

42. Hsieh YL, Chou LW, Hong SF, Chang FC, Tseng SW, Huang CC, Yang CH, Yang CC, Chiu WF. Laser acupuncture attenuates oxaliplatin-induced peripheral neuropathy in patients with gastrointestinal cancer: A pilot prospective cohort study. Acupunct Med 2016; 34:398-405.

43. Yoon J, Jeon JH, Lee YW, Cho CK, Kwon KR, Shin JE, Sagar S, Wong R, Yoo HS. Sweet bee venom pharmacopuncture for chemotherapy-induced peripheral neuropathy. J Acupunct Meridian Stud 2012; 5:156-165.

44. Lindblad K, Bergkvist L, Johansson AC. Evaluation of the treatment of chronic chemotherapy-induced peripheral neuropathy using long-wave diathermy and interferential currents: A randomized controlled trial. Support Care Cancer 2016; 24:2523-2531.

45. Geiger G, Mikus E, Dertinger H, Rick O. Low frequency magnetic field therapy in patients with cytostatic-induced polyneuropathy: A phase II pilot study. Bioelectromagnetics 2015; 36:251-254.

46. Rick O, von Hehn U, Mikus E, Dertinger $\mathrm{H}$, Geiger $\mathrm{G}$. Magnetic field therapy in patients with cytostatics-induced polyneuropathy: A prospective randomized placebo-controlled phase-III study. Bioelectromagnetics 2017; 38:85-94.

47. Pachman DR, Weisbrod BL, Seisler DK, Barton DL, Fee-Schroeder KC, Smith T), Lachance DH, Liu H, Shelerud RA, Cheville AL, Loprinzi CL. Pilot evaluation of Scrambler therapy for the treatment of chemotherapy-induced peripheral neuropathy. Support Care Cancer 2015; 23:943-951.

48. Coyne PJ, Wan W, Dodson P, Swainey C, Smith TJ. A trial of Scrambler therapy in the treatment of cancer pain syndromes and chronic chemotherapy-induced peripheral neuropathy. J Pain Palliat Care Pharmacother 2013; 27:359-364.

49. Smith TJ, Coyne PJ, Parker GL, Dodson P, Ramakrishnan V. Pilot trial of a patient-specific cutaneous electrostimulation device (MC5-A Calmare(R)) for chemotherapy-induced peripheral neuropathy. J Pain Symptom Manage 2010; 40:883-891. 
50. Prinsloo S, Novy D, Driver L, Lyle R, Ramondetta L, Eng C, McQuade J, Lopez $\mathrm{G}$, Cohen L. Randomized controlled trial of neurofeedback on chemotherapyinduced peripheral neuropathy: A pilot study. Cancer 2017; 123:1989-1997.

51. Sacco J, Baas W, Barnes MA, Luberto C, Talat R, Cotton S. The efficacy of percutaneous auricular neurostimulation for chemotherapy-induced peripheral neuropathy: A retrospective chart review. Medical Acupuncture 2016; 28:131-136.

52. Wong R, Major P, Sagar S. Phase 2 study of acupuncture-like transcutaneous nerve stimulation for chemotherapyinduced peripheral neuropathy. Integr Cancer Ther 2016; 15:153-164.

53. Cavaletti G, Frigeni B, Lanzani F, Mattavelli L, Susani E, Alberti P, Cortinovis D, Bidoli P. Chemotherapy-Induced peripheral neurotoxicity assessment: $A$ critical revision of the currently available tools. Eur J Cancer 2010; 46:479-494.

54. McCrary JM, Goldstein D, Boyle F, Cox K, Grimison P, Kiernan MC, Krishnan $\mathrm{AV}$, Lewis CR, Webber K, Baron-Hay S, Horvath L, Park SB. Optimal clinical assessment strategies for chemotherapyinduced peripheral neuropathy (CIPN): A systematic review and Delphi survey. Support Care Cancer 2017; 25:3485-3493.

55. Smith EM, Cohen JA, Pett MA, Beck SL. The reliability and validity of a modified total neuropathy score-reduced and neuropathic pain severity items when used to measure chemotherapy-induced peripheral neuropathy in patients receiving taxanes and platinums. Cancer Nurs 2010; 33:173-183.

56. Cornblath DR, Chaudhry V, Carter K, Lee D, Seysedadr M, Miernicki M, Joh T. Total neuropathy score: Validation and reliability study. Neurology 1999; 53:1660-1664.

57. Cavaletti G, Cornblath DR, Merkies IS Postma TJ, Rossi E, Frigeni B, Alberti P, Bruna J, Velasco R, Argyriou AA, Kalofonos HP, Psimaras D, Ricard D, Pace A, Galie E, Briani C, Dalla Torre C, Faber CG, Lalisang RI, Boogerd W, Brandsma D, Koeppen S, Hense J, Storey D, Kerrigan S, Schenone A, Fabbri S, Valsecchi MG. The chemotherapy-induced peripheral neuropathy outcome measures standardization study: From consensus to the first validity and reliability findings. Ann Oncol 2013; 24:454-462.

58. Hall KE, Liu J, Sima AA, Wiley JW. Impaired inhibitory G-protein function contributes to increased calcium currents in rats with diabetic neuropathy. J Neurophysiol 2001; 86:760-770.

59. Verma $V$, Singh N, Singh Jaggi A. Pregabalin in neuropathic pain: Evidences and possible mechanisms. Curr Neuropharmacol 2014; 12:44-56.

6o. Zilliox L, Russell JW. Treatment of diabetic sensory polyneuropathy. Curr Treat Options Neurol 2011; 13:143-159.

61. Ziegler D, Schneider E, Boess FG, Berggren L, Birklein F. Impact of comorbidities on pharmacotherapy of painful diabetic neuropathy in clinical practice. J Diabetes Complications 2014; 28:698-704.

62. Chaudhry V, Cornblath DR, Polydefkis $\mathrm{M}$, Ferguson A, Borrello I. Characteristics of bortezomib- and thalidomide-induced peripheral neuropathy. J Peripher Nerv Syst 2008; 13:275-282.

63. Kirchmair R, Tietz AB, Panagiotou E, Walter DH, Silver M, Yoon YS, Schratzberger P, Weber A, Kusano K, Weinberg DH, Ropper AH, Isner JM, Losordo DW.
Therapeutic angiogenesis inhibits or rescues chemotherapy-induced peripheral neuropathy: Taxol- and thalidomide-induced injury of vasa nervorum is ameliorated by VEGF. Mol Ther 2007; 15:69-75.

64. Kerckhove N, Collin A, Conde S, Chaleteix C, Pezet D, Balayssac D. Longterm effects, pathophysiological mechanisms, and risk factors of chemotherapy-induced peripheral neuropathies: A comprehensive literature review. Front Pharmacol 2017; 8:86.

65. Johnstone TC, Park GY, Lippard SJ. Understanding and improving platinum anticancer drugs--phenanthriplatin. Anticancer Res 2014; 34:471-476.

66. Addington J, Freimer M. Chemotherapy-induced peripheral neuropathy: An update on the current understanding. F1000Res 2016:5.

67. Sittl R, Lampert A, Huth T, Schuy ET, Link AS, Fleckenstein J, Alzheimer C, Grafe P, Carr RW. Anticancer drug oxaliplatin induces acute cooling-aggravated neuropathy via sodium channel subtype $\mathrm{Na}(\mathrm{V}) \mathrm{1}$.6-resurgent and persistent current. Proc Natl Acad Sci U S A 2012; 109:6704-6709.

68. Jordan MA, Wilson L. Microtubules as a target for anticancer drugs. Nat Rev Cancer 2004; 4:253-265.

69. Gornstein E, Schwarz TL. The paradox of paclitaxel neurotoxicity: Mechanisms and unanswered questions. Neuropharmacology 2014; 76 Pt A:175-183.

70. Geisler S, Doan RA, Strickland A, Huang X, Milbrandt J, DiAntonio A. Prevention of vincristine-induced peripheral neuropathy by genetic deletion of SARM in mice. Brain 2016; 139:3092-3108. 